\title{
Ameliorative Effect of Vitamin C against Monosodium Glutamate Induced Toxicity on Testes and Cauda Epididymal Sperm Reserves in Adult Male Albino Rats
}

\author{
Enas Ibraheem El-Maddah, Mona Mohamed Abo El-Noor' ${ }^{1}$ and Mona Abd El- \\ Haq Abd El-Azeem²
}

\footnotetext{
${ }^{1}$ Department of Forensic Medicine and Clinical Toxicology

${ }^{2}$ Department of Pathology
}

Faculty of Medicine, Tanta University, Tanta, Egypt.

\begin{abstract}
Background: Monosodium glutamate (MSG) is a commonly used food enhancer which causes a wide range of endocrinal disorders including reduced fertility rate in both sexes. Earlier studies have shown that chronic administration of MSG induces oxidative stress in some organs of experimental animals. Accordingly, this work was carried out to study the possible ameliorative effect of vitamin $\mathrm{C}$ on monosodium glutamate induced testicular toxicity in adult male albino rats.

Study design: Sixty adult male albino rats were included in this study and divided into four main groups. Group I used as control group (10 rats). Group II (10 rats) received 100mg/kg/day vitamin C. Group III (20 rats) was subdivided into two subgroups (10 rats each): 100mg/kg/day and $4 \mathrm{gm} / \mathrm{kg} / \mathrm{day}$ MSG treated rats. Group IV (20 rats) was also subdivided into two subgroups and received both MSG and vitamin $\mathrm{C}$ in their previous doses. All animals were treated orally by gastric tube for two months. Body weight, serum testosterone level, cauda epididymal sperm reserves, histopathological examination using H\&E stain and survivin immunostaining were studied.

Results: There was a significant increase in the body weight $(\mathrm{p}<0.05)$ and in the percentage of sperm abnormal forms $(\mathrm{p}<0.05)$, while a significant reduction in the sperm count and motility $(\mathrm{p}<0.05)$ was noticed. These results were dose dependent. Animals treated with $4 \mathrm{gm} / \mathrm{kg}$ MSG showed severely damaged seminiferous tubules with few primary spermatocytes in H\&E stained sections and marked reduction in survivin expression in histochemical study compared to other groups. Moreover, there was significant improvement in all studied parameters on administration of vitamin $\mathrm{C}$ concomitant with MSG which was more observed with low MSG dose $(100 \mathrm{mg} / \mathrm{kg})$. Thus, vitamin C holds a promise as an agent that can reduce MSG induced toxic effects in testes.
\end{abstract}

\section{Introduction}

1 variety of environmental chemicals, industrial pollutants and food additives have been implicated as causing harmful effects (Moore, 2003). Most food additives act either as preservatives, or enhancer of palatability. One such food additive is monosodium glutamate (MSG) which is one of the most flavor enhancers commonly used all over the world by millions of people in their food (Farombi and Onyema, 2006 and Eweka and Om'Iniabohs, 2011). Unfortunately, many Egyptians consume MSG in both ready and home-made food (Swelim, 2004).
Monosodium glutamate (MSG), a white crystalline powder, is the sodium salt of a naturally occurring non-essential amino acid, glutamic acid (Furst and Stehle, 2004). It triggers the taste bud sensitive to 'UMAMI' taste which is referred as the fifth primary taste (Kurihara and Kashiwayanagi, 2000). Umami can be translated "pleasant savory taste". People taste Umami via receptors for glutamate, commonly found in its salt form. The Umami taste often acts to improve or enhance the acceptance response to a variety of food (Bellisle, 2008). MSG is present in: flavored chips and snacks, soups or sauces (canned, packed), prepared meals, frozen foods and 
meals, fresh sausages, marinated meats, stuffed or seasoned chicken, bottled soy or oriental sauces, manufactured meats, luncheon chicken and turkey, flavored tuna, vegetarian burgers and sausages (Bojanic et al., 2009).

The Food and Drug Administration (FDA) of the United States reported that monosodium glutamate is harmless and that it should be kept on the "Generally Recognized as Safe" (GRAS)-list of foods (Eweka and Om'Iniabohs, 2011). As a consequence, it requires no specified daily intake, or an upper limit intake requirement (Moore, 2003).However, inadvertent abuse of this food additive may occur because of its abundance, mostly without labeling, in many food ingredients (Egbuonu et al., 2009).

Some studies indicated that MSG could exhibit harmful effects on human body including: obesity with affected locomotor activity ( Egbuonu et al., 2010), altered lipid metabolism (Egbuonu and Osakwe, 2011), asthma (Scopp, 1991 and Ehlers et al., 1998), epilepsy, stroke and depression (Samuels, 1999 and Mozes and Sefcikova, 2004).

More recent reports have confirmed the adverse effects of MSG on the hypothalamic-pituitary axis of the brain, including neuroexcitatatory/neuroendocrine effects (Feldman and Weidenfeld, 2005 and Seo et al., 2010).

Several studies were done to observe the effects of MSG on the organs concerned with the reproduction as testis, ovary, uterus etc. in the neonatal animals (rats \& mice), fertility rate has been reported to be reduced in both sexes (Mohamed, 2012).

More recent studies have examined the induction of oxidative stress in different tissues of experimental animals after administration of chronic doses of MSG (Singh et al., 2003; Diniz et al., 2004; Farombi and Onyema, 2006 and Onyema et al., 2006).

Vitamin C (ascorbic acid) which is a watersoluble micronutrient, well absorbed by the gastrointestinal tract has a considerable antioxidant activity: it scavenges reactive oxygen species and may, thereby, prevent oxidative damage to the important biological macromolecules, such as DNA, proteins, and lipids (Konopacka, 2004).

Thus, this study aimed to investigate the possible toxic effect of MSG in different doses, as a natural constituent of many food items, on the testes and cauda epididymal sperm reserves of adult male albino rats and to evaluate the possible efficiency of vitamin $\mathrm{C}$, as a natural available cheap antioxidant, in ameliorating such toxicity.

\section{Material and methods}

\section{Chemicals}

Monosodium glutamate was obtained in the form of LGlutamic acid Sodium Salt 500 gm (Eastrin Fine Chemicals. LTD).

Vitamin C was obtained in the form of Lascorbic acid $100 \mathrm{gm}$ (Universal Fine Chemicals PVT. LTD India A.R. grade).

\section{Animals}

The present study was carried out on 60 adult male albino rats, weighing (150- $200 \mathrm{gm}$ ). They were housed in clean properly ventilated cages under the same environmental conditions with free access to food and water throughout the whole period of the experiment. The room temperature was about $22-24{ }^{\circ} \mathrm{C}$ and the animals were exposed to $12: 12$ hours light dark cycles.

\section{Experimental design}

This current work was carried out in Forensic Medicine \& Clinical Toxicology and Pathology Departments in accordance to the guidelines of the Ethical Committee of Medical Research, Faculty of Medicine, Tanta University, Egypt.

After one week of acclimatization, the animals were divided into four main groups:

- Group I: (control group): included 10 animals that further subdivided into two subgroups (five rats each)

○ Subgroup (i): received no treatment.

○ Subgroup (ii): received 0.2 $\mathrm{ml}$ of distilled water (the diluting vehicle for MSG and vitamin $\mathrm{C}$ ).

- Group II: (Vitamin C treated animals): included 10 animals that received vitamin $\mathrm{C}$ in a dose of 100 $\mathrm{mg} / \mathrm{kg}$ /day (Obochi et al., 2009), prepared as $20 \mathrm{mg} / 0.2 \mathrm{ml}$ solution in distilled water.

- Group III: (MSG treated animals): included 20 rats that further subdivided into two subgroups (ten rats each)

○ Subgroup (i): received MSG in a dose of 100 $\mathrm{mg} / \mathrm{kg} /$ day (Obochi et al., 2009), prepared as $20 \mathrm{mg} / 0.2 \mathrm{ml}$ solution in distilled water. The average human daily intake of MSG is estimated to be $0.3-1.0$ $\mathrm{gm}$ in industrialized countries (Geha et al., 
2000). MSG in a dose of $100 \mathrm{mg} / \mathrm{kg} /$ day used in this group was equal to the average intake in human. It was calculated by the use of the formula of dose translation based on body surface area (Reagan- Shaw et al., 2008).

- Subgroup (ii): received MSG in a dose of 4 $\mathrm{gm} / \mathrm{kg} / \mathrm{day}$ (Monno et al., 1995), prepared as $800 \mathrm{mg} / 4 \mathrm{ml}$ solution in distilled water. This MSG dose has been widely used in studying MSG-induced toxic reactions in experimental animals (Diniz et al., 2004 and Farombi and Onyema, 2006). Moreover, this dose is $1 / 4$ LD50 in rats as LD50 in rats and mice is $15,000-18,000 \mathrm{mg} / \mathrm{kg}$ body weight (Walker and Lupien, 2000).

- Group IV: (Protective group): included 20 rats that were further subdivided into two subgroups (ten rats each):

- Each subgroup received vitamin C concomitantly with MSG (at the same dose and duration of their correspondents).

- MSG, vitamin C and their vehicle were given orally by gastric tube once daily for two months.

\section{Methods}

\section{1- Body weight}

Body weight was recorded at the beginning of the experiment of all studied groups and after two months.

\section{2- Hormonal assay}

Prior to sacrificing each rat, $2 \mathrm{ml}$ of blood was collected from the tail vein into a test tube and allowed to clot. The serum was obtained by centrifugation and kept frozen at $-4^{0} \mathrm{C}$ for performing testosterone assay. This assay was carried out using ELISA technique.

The animals were anaesthetized with diethyl ether inhalation and afterward sacrificed by cervical dislocation.

\section{3- Tissue Processing}

- For histological examination: Orchidectomy was performed by open castration method. A midline or pre-scrotal incision was made and the testicles were milked out of the incision site. The testicles were exposed by incising the tunica vaginalis. The spermatic cord was exposed, ligated and incised. The testes were fixed by immersion in Bouin's fluid for 48 hours and processed for paraffin sections of 5 $\mu$ thickness. Sections were stained with hematoxylin and eosin for light microscopy (Bancroft and Gamble, 2002). Caudae epididymes from each animal were also dissected for the study of sperm abnormalities.

- For immunohistochemistry, Four micrometer thick sections of formalin-fixed, paraffinembedded tissue were immuno-stained for survivin (antiapoptotic). By using streptavidin-biotin peroxidase complex method, immunohistochemical staining was performed using the primary antibody for survivin (Cat. \#RB-9245-R7, rabbit polyclonal antibody, Lab Vision/NeoMarkers, Fremont, CA, USA).The paraffin-embedded sections were dewaxed and rehydrated in ethanol. Endogenous peroxidase activity was blocked by incubation with $\mathrm{H}_{2} \mathrm{O}_{2}$ /phosphate buffered saline at room temperature. Antigen retrieval was carried out by microwave heating of tissue sections in $10 \mathrm{mM}$ citrate buffer, pH 6.0 for 15 minutes followed by cooling at room temperature for $20 \mathrm{~min}$. Next, slides were incubated overnight at $4^{\circ} \mathrm{C}$ with the primary antibody after which they were detected using the Envision plus System (Dako). The immunoreaction was developed with diaminobenzidine (DAB; Dako) for 5 min, and then counterstained with hematoxylin. For the negative control, the primary antibody was replaced by a phosphate buffer solution, whereas the other steps of the procedure were the same. The cellular labeling of survivin was visualized in the nucleus and the cytoplasm as brown coloration (Lechler et al., 2007).

\section{4- Semen examination}

Semen examination of all groups was performed according to Bearden and Fuquay, (1997) as following:

The epididymis was dissected and its content was obtained by cutting the epididymis and squeezing its content into one centimeter saline in a clean Petri dish. The aliquot was subjected to the following: 
A. Progressive sperm motility: A small droplet of semen was placed on a clean slide and covered with a cover slip and then several fields were examined under microscope and the number of progressively motile sperms per 100 sperms was estimated and recorded.

B. Sperm cell concentration: The sperm cell concentration was estimated by direct cell count using a hemocytometer slide after diluting semen to the desired concentration. The cover slip was placed over the counting chamber and a small amount of diluted semen is placed at the edge of the cover slip then the slide is placed under the microscope to count the number of the sperm in the four corners and the central square, then, the number of the sperm per epididymis was counted using the following formula:

No. of sperms /epididymis (million) $=$ number of sperm $\mathrm{x}$ dilution rate $\mathrm{x}$ 50,000

C. Epididymal sperm abnormalities: A drop of epididymal content of each rat was mixed with equal drops of Eosine $1 \%$ and nigrosine $5 \%$ stain and carefully mixed, two dry clean slides were used to make the smear and the stained slide was allowed to dry and examined under high power of microscope $(\mathrm{x} 40)$. The percentage of abnormal sperms was calculated as mean from counting 100 sperm in each of the two slides. Photomicrographs of the desired sections were made for further observations.

\section{5- Morphometric study}

The morphometric measurements were carried out using the Leica Qwin 500 (Leica, London, UK) image analyzer computer system (Central Laboratory, Faculty of Medicine, Tanta University). Ten high-power fields, from each slide of all animals of each group, were used to evaluate the immunohistochemistry results by counting survivin positively stained cells. Immunoreactivity was assigned one of the following four categories: $0(<20 \%$ of cells show staining); +1 , (20$50 \%)$; $+2,(50-75 \%)$; and $+3,(>75 \%)$. Immunoreactivity in more than $20 \%$ of cells was considered positive i.e. score equal to or above +1 (Kennedy et al., 2003).

\section{Statistics}

Data were expressed as means \pm standard deviation. ANOVA test was used for comparison among different groups in quantitative data. Scheffe test was used to compare each group versus another. $\mathrm{P}<0.05$ was considered significant.

\section{Results}

\section{A. Initial and final body weights}

Table (1) and figure (1) show mean values of the initial and final body weights of adult male rats of all studied groups. There was no significant difference of the mean values of the initial body weight between all the studied groups. At the end of the experiment, the mean values of the final body weight in MSG- treated rats (group III) were increased significantly $(\mathrm{P}<0.05)$ compared with other studied groups (I, II, IV), while treatment with vitamin $\mathrm{C}$ along with MSG in group IV significantly reduced this increase.

The mean values of the final body weight in $4 \mathrm{gm} / \mathrm{kg}$ MSG- treated rats (326.4 \pm 13.81$)$ were significantly higher than those of $100 \mathrm{mg} / \mathrm{kg}$ MSGtreated rats $(287.6 \pm 13.86)$. There was also significant decrease in the final body weight in $100 \mathrm{mg} / \mathrm{kg} \mathrm{MSG-}$ vitamin $\mathrm{C}$ treated animals $(252.5 \pm 10.61)$ compared to $4 \mathrm{gm} / \mathrm{kg} \quad$ MSG-vitamin $\mathrm{C}$ treated animals (265.2 \pm 14.10$)$.

\section{B. Serum testosterone levels}

Table (2) and figure (2) show mean values of serum testosterone levels $(\mathrm{ng} / / \mathrm{ml})$ in adult male rats of all studied groups.

There was significant decrease $(\mathrm{P}<0.05)$ in mean values of serum testosterone levels in MSG- treated animals (group III) compared with other studied groups (I, II, IV), while treatment with vitamin $\mathrm{C}$ along with MSG in group IV significantly improved this reduction. The mean values of serum testosterone levels in $4 \mathrm{gm} / \mathrm{kg}$ MSG- treated rats $(0.15 \pm 0.031)$ were significantly decreased compared to those in $100 \mathrm{mg} / \mathrm{kg}$ MSG- treated rats $(0.32 \pm 0.047)$.

There was significant improvement in $100 \mathrm{mg} / \mathrm{kg}$ MSG-vitamin $\mathrm{C}$ treated animals (0.94 \pm 0.036$)$ compared to $4 \mathrm{gm} / \mathrm{kg}$ MSG-vitamin C treated animals $(0.83 \pm 0.020)$.

\section{Assessment of caudaepididymal sperm reserves}

Table (3) and figure (3) Show mean values of cauda epididymal sperm reserves as regard sperm count $\left(\mathrm{X} 10^{6}\right)$, sperm motility $(\%)$ and incidence of abnormal forms $(\%)$. 
The sperm count $\left(\mathrm{X10}^{6}\right)$ showed high significant decrease $(\mathrm{P}<0.05)$ in rats on $\mathrm{MSG}$ alone (group III) compared with other studied groups (I, II, IV), while treatment with vitamin $\mathrm{C}$ along with MSG in group IV significantly improve this decrease. The mean values of sperm count of $4 \mathrm{gm} / \mathrm{kg}$ MSG-treated rats $(9.30 \pm 1.15)$ were significantly decreased compared to $100 \mathrm{mg} / \mathrm{kg}$ MSG- treated rats $(21.5 \pm 4.1)$. Moreover, sperm count was significantly improved in $100 \mathrm{mg} / \mathrm{kg}$ MSG- vitamin $\mathrm{C}$ treated animals (34.5 \pm 2.91$)$ compared with $4 \mathrm{gm} / \mathrm{kg}$ MSG- vitamin C treated group (26.2 \pm 3.33$)$.

The sperm motility (\%) showed significant decrease $(\mathrm{P}<0.05)$ in rats treated with MSG alone (group III) compared with other studied groups (I.II, IV), while treatment with vitamin $\mathrm{C}$ along with MSG in group IV significantly improve this reduction. The mean values of sperm motility in $4 \mathrm{gm} / \mathrm{kg}$ MSG- treated rats $(29.9 \pm 2.33)$ were significantly decreased compared to $100 \mathrm{mg} / \mathrm{kg}$ MSG- treated rats $(60.4 \pm 3.59)$.

Moreover, there was significant decrease in sperm motility (\%) in $100 \mathrm{mg} / \mathrm{kg}$ MSG-vitamin C treated rats $(81.6 \pm 2.71)$ compared to $4 \mathrm{gm} / \mathrm{kg}$ MSGvitamin $C$ treated group (77.2 \pm 3.25$)$.

The incidence of abnormal forms (\%) showed high significant increase $(\mathrm{P}<0.05)$ in $\mathrm{MSG}$ treated animals (group III) compared with other studied groups (I.II, IV), while treatment with vitamin C along with MSG in group IV significantly reduced this increase. The mean values of abnormal forms of sperms in $4 \mathrm{gm} / \mathrm{kg}$ MSG- treated group (38 \pm 2.16 ) were significantly increased compared to $100 \mathrm{mg} / \mathrm{kg}$ MSGtreated group (24.9 \pm 3.84$)$.

Moreover, sperm count was significantly reduced in $100 \mathrm{mg} / \mathrm{kg}$ MSG-vitamin $\mathrm{C}$ treated rats (6.50 \pm 0.84$)$ compared with $4 \mathrm{gm} / \mathrm{kg}$ MSG-vitamin C treated group (11.2 \pm 1.36$)$.

\section{Histopathological results}

Figure (4) showed some of these abnormal sperm forms compared to the normal morphology of sperm in the form of normal head shape with characterized hook and straight tail (A). They include sperms with bent necks (B), bent tails (C) or bent neck and tail (D).

$\mathrm{H} \& \mathrm{E}$ stained sections of the testes in adult male rats of the control and vitamin $\mathrm{C}$ groups (group I,II) showed normal morphology of the testis where there was normal testicular histology and normal spermatogenic progression with active seminiferous tubules and interstitial spaces (figures 5,6).

MSG treated rats with $100 \mathrm{~m} / \mathrm{kg}$ were less affected than those treated with $4 \mathrm{gm} / \mathrm{kg}$ as illustrated in figures $(7,8)$. There was damage of spermatogonia and elongation of primary spermatocytes with few damaged late spermatids in the tubules in $100 \mathrm{mg} / \mathrm{kg}$ MSG-treated animals (figure 7), while in $4 \mathrm{gm} / \mathrm{kg} \mathrm{MSG-}$ treated group there was severely damaged seminiferous tubules with few primary spermatocytes in the tubules(figure 8).

As regards to the MSG-vitamin $\mathrm{C}$ treated rats, there was marked improvement in $100 \mathrm{mg} / \mathrm{kg}$ MSGvitamin $C$ treated animals as illustrated in figure (9) in which there was some normal seminiferous tubules with normal spermatogensis admixed with degenerated tubules. While vitamin $\mathrm{C}$ with $4 \mathrm{gm} / \mathrm{kg}$ MSG- treated animals was less effective as demonstrated in figure (10) where there was few preserved normal seminiferous tubules with normal spermatogensis admixed with severely damaged tubules.

\section{Immunohistochemical results}

The sections of the control and vitamin $\mathrm{C}$ treated animals (group I,II) showed that spermatogenic cells had a positive survivin reaction in the nuclei and the cytoplasm. Survivin immunostaining showed strong expression in most of the nuclei and cytoplasm of spermatogenic cells of the control and vitamin C treated animals (figure 11).

As regards to MSG-treated rats with $100 \mathrm{mg} / \mathrm{kg}$, there was mild nuclear and cytoplasmic expression in spermatogenic cells of most seminiferous tubules (figure 12), while $4 \mathrm{gm} / \mathrm{kg}$ MSG-treated animals showed marked reduction in survivin expression in spermatogenic cells of most seminiferous tubules (figure 13).

Concerning the group treated with vitamin $\mathrm{C}$ along with MSG (group IV), there was strong effect of vitamin $\mathrm{C}$ on rats treated with $100 \mathrm{mg} / \mathrm{kg}$ as illustrated in figure (14) in which there was strong nuclear and cytoplasmic survivin immunostaining of the spermatogenic cells, while moderate nuclear and cytoplasmic surviving immunostaining of spermatogenic cells was found in $4 \mathrm{gm} / \mathrm{kg}$ MSGvitamin $\mathrm{C}$ treated animals (figure 15).

Table (4) and figure (16) show significant decrease in survivin expression in rats on MSG alone (group III) compared with other studied groups (I, II, IV), while treatment with vitamin $\mathrm{C}$ along with MSG in group IV significantly improved this decrease. There was no significant difference between $100 \mathrm{mg} / \mathrm{kg}$ MSG and $4 \mathrm{gm} / \mathrm{kg}$ MSG- treated animals. Moreover, survivin expression was significantly improved in $100 \mathrm{mg} / \mathrm{kg}$ MSG- vitamin $\mathrm{C}$ treated animals $(2.6 \pm 0.63)$ compared with $4 \mathrm{gm} / \mathrm{kg}$ MSG- vitamin C treated group ( $2 \pm 0.42)$. 
Table (1): ANOVA one way statistical analysis of initial and final body weights (gm) among all studied groups

\begin{tabular}{|c|c|c|c|c|c|c|c|c|}
\hline & \multicolumn{2}{|c|}{ GI (control) } & \multirow{2}{*}{$\begin{array}{c}\text { GII } \\
\begin{array}{c}\text { Vitamin } \\
\text { C }\end{array}\end{array}$} & \multicolumn{2}{|c|}{ GIII } & \multicolumn{2}{|c|}{ GIV } \\
\hline & & Negative & Positive & & $\begin{array}{c}\text { MSG } \\
100 \mathrm{mg} / \mathrm{kg}\end{array}$ & $\begin{array}{c}\text { MSG } \\
4 \mathrm{gm} / \mathrm{kg}\end{array}$ & $\begin{array}{c}\text { MSG } \\
\text { 100mg/kg } \\
+ \text { vitamin } \\
\text { C }\end{array}$ & $\begin{array}{c}\text { MSG } \\
4 \mathrm{gm} / \mathrm{kg} \\
+ \\
\text { vitamin } \\
\mathrm{C}\end{array}$ \\
\hline \multicolumn{2}{|l|}{$\mathrm{NO}$} & 5 & 5 & 10 & 10 & 10 & 10 & 10 \\
\hline \multirow{4}{*}{$\begin{array}{l}\text { Initial } \\
\text { B.M (gm) }\end{array}$} & Mean & 168 & 167.2 & 171.7 & 167.6 & 168.9 & 170.6 & 172.3 \\
\hline & $+\mathrm{SD}$ & 11.49 & 19.72 & 15.77 & 13.67 & 14.83 & 10.96 & 14.55 \\
\hline & F. & \multicolumn{7}{|c|}{0.160} \\
\hline & $\mathrm{P}$ & \multicolumn{7}{|c|}{0.696} \\
\hline \multicolumn{9}{|c|}{ Sheffe test } \\
\hline $\begin{array}{l}\text { Control\& } \\
\text { vitamin C }\end{array}$ & $\begin{array}{l}\text { Control\& } \\
\text { MSG } \\
100 \mathrm{mg} / \mathrm{kg}\end{array}$ & $\begin{array}{l}\text { Control\& } \\
\mathrm{MSG} \\
4 \mathrm{gm} / \mathrm{kg}\end{array}$ & $\begin{array}{l}\text { Control\& MSG } \\
100 \mathrm{mg} / \mathrm{kg}+ \\
\text { vitamin C }\end{array}$ & $\begin{array}{l}\text { Control\& } \\
\mathrm{MSG} \\
4 \mathrm{gm} / \mathrm{kg} \\
+ \\
\text { vitamin } \\
\mathrm{C}\end{array}$ & $\begin{array}{l}\text { vitamin } \\
\text { C\& MSG } \\
100 \mathrm{mg} / \mathrm{kg}\end{array}$ & $\begin{array}{l}\text { vitamin } \\
\mathrm{C} \& \\
\mathrm{MSG} \\
4 \mathrm{gm} / \mathrm{kg}\end{array}$ & $\begin{array}{l}\text { vitamin } \\
\mathrm{C} \& \mathrm{MSG} \\
100 \mathrm{mg} / \mathrm{kg} \\
+ \text { vitamin } \\
\mathrm{C}\end{array}$ & $\begin{array}{l}\text { vitamin } \\
\text { C\& } \\
\mathrm{MSG} \\
4 \mathrm{gm} / \mathrm{kg} \\
+ \\
\text { vitamin } \\
\mathrm{C}\end{array}$ \\
\hline 0.715 & 0.880 & 0.960 & 0.821 & 0.660 & 0.528 & 0.662 & 0.865 & 0.663 \\
\hline $\begin{array}{l}\mathrm{MSG} \\
100 \mathrm{mg} / \mathrm{kg} \\
\& \mathrm{MSG} 4 \\
\mathrm{gm} / \mathrm{kg}\end{array}$ & $\begin{array}{l}\text { MSG } \\
100 \mathrm{mg} / \mathrm{kg} \& \\
\mathrm{MSG} \\
100 \mathrm{mg} / \mathrm{kg} \\
+ \text { vitamin C }\end{array}$ & $\begin{array}{l}\mathrm{MSG} \\
4 \mathrm{gm} / \mathrm{kg} \& \\
\mathrm{MSG} \\
4 \mathrm{gm} / \mathrm{kg} \\
+ \\
\text { vitamin } \\
\mathrm{C} \\
\end{array}$ & $\begin{array}{l}\mathrm{MSG} 100 \mathrm{mg} / \mathrm{kg}+ \\
\text { vitamin C\& MSG } \\
4 \mathrm{gm} / \mathrm{kg}+\text { vitamin C }\end{array}$ & & & & & \\
\hline 0.841 & 0.644 & 0.600 & 0.793 & & & & & \\
\hline \multirow{4}{*}{$\begin{array}{l}\text { Final } \\
\text { B.M (gm) }\end{array}$} & Mean & 248.2 & 247.2 & 251.4 & 287.6 & 326.4 & 252.5 & 265.2 \\
\hline & $+\mathrm{SD}$ & 11.77 & 19.72 & 15.19 & 13.86 & 13.81 & 10.61 & 14.10 \\
\hline & F. & \multicolumn{7}{|c|}{38.669} \\
\hline & $\mathrm{P}$ & \multicolumn{7}{|c|}{$0.001 * *$} \\
\hline \multicolumn{9}{|c|}{ Sheffe test } \\
\hline $\begin{array}{l}\text { Control } \\
\& \\
\text { vitamin C }\end{array}$ & $\begin{array}{l}\text { Control\& } \\
\text { MSG } \\
100 \mathrm{mg} / \mathrm{kg}\end{array}$ & $\begin{array}{l}\text { Control\& } \\
\mathrm{MSG} \\
4 \mathrm{gm} / \mathrm{kg}\end{array}$ & $\begin{array}{l}\text { Control\& MSG } \\
100 \mathrm{mg} / \mathrm{kg}+ \\
\text { vitamin C }\end{array}$ & $\begin{array}{l}\text { Control\& } \\
\mathrm{MSG} \\
4 \mathrm{gm} / \mathrm{kg} \\
+ \\
\text { vitamin } \\
\mathrm{C}\end{array}$ & $\begin{array}{l}\text { vitamin } \\
\text { C\& MSG } \\
100 \mathrm{mg} / \mathrm{kg}\end{array}$ & $\begin{array}{l}\text { vitamin } \\
\mathrm{C} \& \\
\mathrm{MSG} \\
4 \mathrm{gm} / \mathrm{kg}\end{array}$ & $\begin{array}{l}\text { vitamin } \\
\mathrm{C} \& \mathrm{MSG} \\
100 \mathrm{mg} / \mathrm{kg} \\
+ \text { vitamin } \\
\mathrm{C}\end{array}$ & $\begin{array}{l}\text { vitamin } \\
\mathrm{C} \& \\
\mathrm{MSG} \\
4 \mathrm{gm} / \mathrm{kg} \\
+ \\
\text { vitamin } \\
\mathrm{C}\end{array}$ \\
\hline 0.679 & $0.001 * *$ & $0.001 * *$ & 0.579 & 0.113 & $0.001 * *$ & $0.001 * *$ & 0.862 & 0.072 \\
\hline $\begin{array}{l}\text { MSG } \\
100 \mathrm{mg} / \mathrm{kg} \\
\& \mathrm{MSG} \\
4 \mathrm{gm} / \mathrm{kg}\end{array}$ & $\begin{array}{l}\text { MSG } \\
100 \mathrm{mg} / \mathrm{kg} \& \\
\mathrm{MSG} \\
100 \mathrm{mg} / \mathrm{kg} \\
+ \text { vitamin C }\end{array}$ & $\begin{array}{l}\text { MSG } \\
4 \mathrm{gm} / \mathrm{kg} \& \\
\mathrm{MSG} \\
4 \mathrm{gm} / \mathrm{kg} \\
+ \\
\text { vitamin } \\
\mathrm{C}\end{array}$ & $\begin{array}{l}\mathrm{MSG} 100 \mathrm{mg} / \mathrm{kg}+ \\
\text { vitamin C\& MSG } \\
4 \mathrm{gm} / \mathrm{kg}+\text { vitamin C }\end{array}$ & & & & & \\
\hline $0.001 * *$ & $0.001 * *$ & $0.001 * *$ & $0.003 *$ & & & & & \\
\hline
\end{tabular}

$N O=$ number, B.M= body weight; $* p<0.05$ significant; $* * p<0.001$ highly significant. 
Table (2): ANOVA one way statistical analysis of serum testosterone levels (ng/ml) among all studied groups.

\begin{tabular}{|c|c|c|c|c|c|c|c|c|}
\hline & \multicolumn{2}{|c|}{ GI (control) } & \multirow{2}{*}{$\begin{array}{c}\text { GII } \\
\text { Vitami } \\
\text { n C }\end{array}$} & \multicolumn{2}{|c|}{ GIII } & \multicolumn{2}{|c|}{ GIV } \\
\hline & & Negative & Positive & & $\begin{array}{c}\text { MSG } \\
100 \mathrm{mg} / \mathrm{k} \\
\mathrm{g}\end{array}$ & $\begin{array}{c}\text { MSG } \\
4 \mathrm{gm} / \mathrm{kg}\end{array}$ & $\begin{array}{c}\text { MSG } \\
\text { 100mg/kg } \\
+ \\
\text { Vitamin } \\
\text { C }\end{array}$ & $\begin{array}{c}\text { MSG } \\
4 \mathrm{gm} / \mathrm{kg} \\
+ \\
\text { Vitami } \\
\text { n C }\end{array}$ \\
\hline \multicolumn{2}{|l|}{$\mathrm{NO}$} & 5 & 5 & 10 & 10 & 10 & 10 & 10 \\
\hline \multirow{4}{*}{$\begin{array}{l}\text { Testosterone } \\
\text { (ng/ml) }\end{array}$} & Mean & 0.95 & 0.95 & 0.95 & 0.32 & 0.15 & 0.94 & 0.83 \\
\hline & $+\mathrm{SD}$ & 0.008 & 0.010 & 0.012 & 0.047 & 0.031 & 0.036 & 0.020 \\
\hline & F. & \multicolumn{7}{|c|}{65.338} \\
\hline & $\mathrm{P}$ & \multicolumn{7}{|c|}{$0.001 * *$} \\
\hline \multicolumn{9}{|c|}{ Sheffe test } \\
\hline $\begin{array}{l}\text { Control\& } \\
\text { vitamin C }\end{array}$ & $\begin{array}{l}\text { Control\& } \\
\text { MSG } \\
100 \mathrm{mg} / \mathrm{kg}\end{array}$ & $\begin{array}{l}\text { Control\& } \\
\mathrm{MSG} \\
4 \mathrm{gm} / \mathrm{kg}\end{array}$ & $\begin{array}{l}\text { Control\& } \\
\mathrm{MSG} \\
100 \mathrm{mg} / \mathrm{k} \\
\mathrm{g}+ \\
\text { vitamin } \\
\mathrm{C}\end{array}$ & $\begin{array}{l}\text { Contro } \\
1 \& \\
\mathrm{MSG} \\
4 \mathrm{gm} / \mathrm{k} \\
\mathrm{g}+ \\
\text { vitami } \\
\mathrm{n} \mathrm{C}\end{array}$ & $\begin{array}{l}\text { vitamin } \\
\mathrm{C} \& \mathrm{MSG} \\
100 \mathrm{mg} / \mathrm{kg}\end{array}$ & $\begin{array}{l}\text { vitamin } \\
\text { C\& } \\
\mathrm{MSG} \\
4 \mathrm{gm} / \mathrm{kg}\end{array}$ & $\begin{array}{l}\text { vitamin } \\
\mathrm{C} \& \mathrm{MSG} \\
100 \mathrm{mg} / \mathrm{kg} \\
+ \text { vitamin } \\
\mathrm{C}\end{array}$ & $\begin{array}{l}\text { vitamin } \\
\mathrm{C} \& \\
\mathrm{MSG} \\
4 \mathrm{gm} / \mathrm{kg} \\
+ \\
\text { vitamin } \\
\mathrm{C}\end{array}$ \\
\hline 0.903 & $0.001 * *$ & $0.001 * *$ & 0.125 & 0.096 & $0.001 * *$ & $0.001 * *$ & 0.152 & 0.336 \\
\hline $\begin{array}{l}\mathrm{MSG} \\
100 \mathrm{mg} / \mathrm{kg} \& \\
\mathrm{MSG} 4 \mathrm{gm} / \mathrm{kg}\end{array}$ & $\begin{array}{l}\text { MSG } \\
100 \mathrm{mg} / \mathrm{kg} \\
\& \mathrm{MSG} \\
100 \mathrm{mg} / \mathrm{kg} \\
+ \text { vitamin } \\
\mathrm{C}\end{array}$ & $\begin{array}{l}\mathrm{MSG} \\
4 \mathrm{gm} / \mathrm{kg} \& \\
\mathrm{MSG} \\
4 \mathrm{gm} / \mathrm{kg}+ \\
\text { vitamin C }\end{array}$ & $\begin{array}{l}\text { MSG } \\
100 \mathrm{mg} / \mathrm{k} \\
\mathrm{g}+ \\
\text { vitamin } \\
\mathrm{C} \& \mathrm{MSG} \\
4 \mathrm{gm} / \mathrm{kg}+ \\
\text { vitamin } \\
\mathrm{C}\end{array}$ & & & & & \\
\hline $0.001 * *$ & $0.001 * *$ & $0.001 * *$ & $0.001 * *$ & & & & & \\
\hline
\end{tabular}

$N O=$ number; $* p<0.05$ significant; $* * p<0.001$ highly significant.

Table (3): ANOVA one way statistical analysis of cauda epididymal sperm reserves, sperm count $\left(\mathrm{X10}^{6}\right)$, sperm motility (\%) and incidence of abnormal forms (\%) among all studied groups.

\begin{tabular}{|c|c|c|c|c|c|c|c|c|}
\hline & \multicolumn{2}{|c|}{ GI (control) } & \multirow[b]{2}{*}{$\begin{array}{c}\text { GII } \\
\text { Vitamin } \\
\text { C }\end{array}$} & \multicolumn{2}{|c|}{ GIII } & \multicolumn{2}{|c|}{ GIV } \\
\hline & & Negative & Positive & & $\begin{array}{c}\text { MSG } \\
100 \mathrm{mg} / \mathrm{kg}\end{array}$ & $\begin{array}{c}\text { MSG } \\
4 \mathrm{gm} / \mathrm{kg}\end{array}$ & $\begin{array}{c}\text { MSG } \\
100 \mathrm{mg} / \mathrm{kg} \\
+ \\
\text { Vitamin } \\
\mathrm{C} \\
\end{array}$ & $\begin{array}{c}\text { MSG } \\
4 \mathrm{gm} / \mathrm{kg} \\
+ \\
\text { Vitamin } \\
\mathrm{C}\end{array}$ \\
\hline \multicolumn{2}{|l|}{$\mathrm{NO}$} & 5 & 5 & 10 & 10 & 10 & 10 & 10 \\
\hline \multirow{4}{*}{$\begin{array}{l}\text { Sperm } \\
\text { count }\left(10^{6}\right)\end{array}$} & Mean & 37.6 & 35.2 & 36 & 21.5 & 9.30 & 34.5 & 26.2 \\
\hline & $\pm \underline{\mathrm{SD}}$ & 3.04 & 1.92 & 2.74 & 4.1 & 1.15 & 2.91 & 3.33 \\
\hline & F. & \multicolumn{7}{|c|}{12.336} \\
\hline & $\mathrm{P}$ & \multicolumn{7}{|c|}{$0.001 * *$} \\
\hline \multicolumn{9}{|c|}{ Sheffe test } \\
\hline $\begin{array}{l}\text { Control\& } \\
\text { vitamin C }\end{array}$ & $\begin{array}{l}\text { Control\& } \\
\text { MSG } \\
100 \mathrm{mg} / \mathrm{kg}\end{array}$ & $\begin{array}{l}\text { Control\& } \\
\text { MSG } \\
4 \mathrm{gm} / \mathrm{kg}\end{array}$ & $\begin{array}{c}\text { Control\& } \\
\mathrm{MSG} \\
100 \mathrm{mg} / \mathrm{kg}+ \\
\text { vitamin C }\end{array}$ & $\begin{array}{c}\text { Control\& } \\
\mathrm{MSG} \\
4 \mathrm{gm} / \mathrm{kg} \\
+ \\
\text { vitamin } \\
\mathrm{C}\end{array}$ & $\begin{array}{c}\text { vitamin } \\
\mathrm{C} \& \mathrm{MSG} \\
100 \mathrm{mg} / \mathrm{kg}\end{array}$ & $\begin{array}{c}\text { vitamin } \\
\mathrm{C} \& \\
\mathrm{MSG} \\
4 \mathrm{gm} / \mathrm{kg}\end{array}$ & $\begin{array}{c}\text { vitamin } \\
\text { C\& } \mathrm{MSG} \\
100 \mathrm{mg} / \mathrm{kg} \\
+ \text { vitamin } \\
\mathrm{C}\end{array}$ & $\begin{array}{c}\text { vitamin } \\
\mathrm{C} \& \\
\mathrm{MSG} \\
4 \mathrm{gm} / \mathrm{kg} \\
+ \\
\text { vitamin } \\
\mathrm{C}\end{array}$ \\
\hline 0.322 & $0.001 * *$ & $0.001 * *$ & 0.058 & 0.095 & $0.001 * *$ & $0.001 * *$ & 0.257 & 0.067 \\
\hline $\begin{array}{c}\mathrm{MSG} \\
100 \mathrm{mg} / \mathrm{kg} \& \\
\mathrm{MSG} \\
4 \mathrm{gm} / \mathrm{kg}\end{array}$ & $\begin{array}{c}\mathrm{MSG} \\
100 \mathrm{mg} / \mathrm{kg} \& \\
\mathrm{MSG} \\
100 \mathrm{mg} / \mathrm{kg} \\
+ \text { vitamin } \mathrm{C} \\
\end{array}$ & $\begin{array}{c}\mathrm{MSG} \\
\text { 4gm/kg\& } \\
\mathrm{MSG} \\
4 \mathrm{gm} / \mathrm{kg}+ \\
\text { vitamin C }\end{array}$ & $\begin{array}{c}\mathrm{MSG} \\
100 \mathrm{mg} / \mathrm{kg}+ \\
\text { vitamin C\& } \\
\mathrm{MSG} 4 \mathrm{gm} / \mathrm{kg} \\
+ \text { vitamin C }\end{array}$ & & & & & \\
\hline $0.001 * *$ & $0.001 * *$ & $0.001 * *$ & $0.001 * *$ & & & & & \\
\hline
\end{tabular}




\begin{tabular}{|c|c|c|c|c|c|c|c|c|}
\hline & & \multicolumn{2}{|c|}{ GI (control) } & \multirow[b]{2}{*}{$\begin{array}{c}\text { GII } \\
\text { Vitamin } \\
\text { C }\end{array}$} & \multicolumn{2}{|c|}{ GIII } & \multicolumn{2}{|c|}{ GIV } \\
\hline & & Negative & Positive & & $\begin{array}{c}\text { MSG } \\
100 \mathrm{mg} / \mathrm{kg}\end{array}$ & $\begin{array}{c}\text { MSG } \\
4 \mathrm{gm} / \mathrm{kg}\end{array}$ & $\begin{array}{c}\text { MSG } \\
100 \mathrm{mg} / \mathrm{kg} \\
+ \\
\text { Vitamin } \\
\text { C }\end{array}$ & $\begin{array}{c}\text { MSG } \\
4 \mathrm{gm} / \mathrm{kg} \\
+ \\
\text { Vitamin } \\
\mathrm{C}\end{array}$ \\
\hline \multirow{4}{*}{$\begin{array}{l}\text { Motility } \\
(\%)\end{array}$} & Mean & 83.4 & 84.6 & 84.1 & 60.4 & 29.9 & 81.6 & 77.2 \\
\hline & $\pm \mathrm{SD}$ & 2.19 & 2.30 & 2.23 & 3.59 & 2.33 & 2.71 & 3.25 \\
\hline & F. & \multicolumn{7}{|c|}{41.225} \\
\hline & $\mathrm{P}$ & \multicolumn{7}{|c|}{$0.001 * *$} \\
\hline \multicolumn{9}{|c|}{ Sheffe test } \\
\hline $\begin{array}{l}\text { Control\& } \\
\text { vitamin C }\end{array}$ & $\begin{array}{c}\text { Control\& } \\
\text { MSG } \\
100 \mathrm{mg} / \mathrm{kg}\end{array}$ & $\begin{array}{l}\text { Control\& } \\
\text { MSG } \\
4 \mathrm{gm} / \mathrm{kg}\end{array}$ & $\begin{array}{c}\text { Control\& } \\
\mathrm{MSG} \\
100 \mathrm{mg} / \mathrm{kg}+ \\
\text { vitamin C }\end{array}$ & $\begin{array}{c}\text { Control\& } \\
\mathrm{MSG} \\
4 \mathrm{gm} / \mathrm{kg} \\
+ \\
\text { vitamin } \\
\mathrm{C}\end{array}$ & $\begin{array}{c}\text { vitamin } \\
\mathrm{C} \& \mathrm{MSG} \\
100 \mathrm{mg} / \mathrm{kg}\end{array}$ & $\begin{array}{c}\text { vitamin } \\
\mathrm{C} \& \\
\mathrm{MSG} \\
4 \mathrm{gm} / \mathrm{kg}\end{array}$ & $\begin{array}{c}\text { vitamin } \\
\mathrm{C} \& \mathrm{MSG} \\
100 \mathrm{mg} / \mathrm{kg} \\
+ \text { vitamin } \\
\mathrm{C}\end{array}$ & $\begin{array}{c}\text { vitamin } \\
\mathrm{C} \& \\
\mathrm{MSG} \\
4 \mathrm{gm} / \mathrm{kg} \\
+ \\
\text { vitamin } \\
\mathrm{C}\end{array}$ \\
\hline 0.673 & $0.001 * *$ & $0.001 * *$ & 0.280 & 0.142 & $0.001 * *$ & $0.001 * *$ & 0.069 & 0.083 \\
\hline $\begin{array}{c}\mathrm{MSG} \\
100 \mathrm{mg} / \mathrm{kg} \& \\
\mathrm{MSG} \\
4 \mathrm{gm} / \mathrm{kg}\end{array}$ & $\begin{array}{c}\mathrm{MSG} \\
100 \mathrm{mg} / \mathrm{kg} \& \\
\mathrm{MSG} \\
100 \mathrm{mg} / \mathrm{kg} \\
+ \text { vitamin C } \\
\end{array}$ & $\begin{array}{c}\mathrm{MSG} \\
\text { 4gm/kg\& } \\
\mathrm{MSG} \\
4 \mathrm{gm} / \mathrm{kg}+ \\
\text { vitamin C }\end{array}$ & $\begin{array}{c}\mathrm{MSG} \\
100 \mathrm{mg} / \mathrm{kg}+ \\
\text { vitamin C\& } \\
\mathrm{MSG} 4 \mathrm{gm} / \mathrm{kg} \\
+ \text { vitamin C }\end{array}$ & & & & & \\
\hline $0.001 * *$ & $0.001 * *$ & $0.001 * *$ & $0.001 * *$ & & & & & \\
\hline \multirow{4}{*}{$\begin{array}{l}\text { Abnormal } \\
\text { forms }(\%)\end{array}$} & Mean & 6 & 5.6 & 5.6 & 24.9 & 38 & 6.50 & 11.2 \\
\hline & $\pm \mathrm{SD}$ & 1.41 & 0.54 & 0.96 & 3.84 & 2.16 & 0.84 & 1.36 \\
\hline & F. & \multicolumn{7}{|c|}{39.663} \\
\hline & $\mathrm{P}$ & \multicolumn{7}{|c|}{$0.001 * *$} \\
\hline \multicolumn{9}{|c|}{$\begin{array}{c}\text { Sheffe test } \\
\end{array}$} \\
\hline $\begin{array}{l}\text { Control\& } \\
\text { vitamin C }\end{array}$ & $\begin{array}{c}\text { Control\& } \\
\text { MSG } \\
100 \mathrm{mg} / \mathrm{kg}\end{array}$ & $\begin{array}{c}\text { Control\& } \\
\text { MSG } \\
4 \mathrm{gm} / \mathrm{kg}\end{array}$ & $\begin{array}{c}\text { Control\& } \\
\mathrm{MSG} \\
100 \mathrm{mg} / \mathrm{kg}+ \\
\text { vitamin C }\end{array}$ & $\begin{array}{c}\text { Control\& } \\
\mathrm{MSG} \\
4 \mathrm{gm} / \mathrm{kg} \\
+ \\
\text { vitamin } \\
\mathrm{C}\end{array}$ & $\begin{array}{c}\text { vitamin } \\
\mathrm{C} \& \mathrm{MSG} \\
100 \mathrm{mg} / \mathrm{kg}\end{array}$ & $\begin{array}{l}\text { vitamin } \\
\mathrm{C} \& \\
\mathrm{MSG} \\
4 \mathrm{gm} / \mathrm{kg}\end{array}$ & $\begin{array}{c}\text { vitamin } \\
\mathrm{C} \& \mathrm{MSG} \\
100 \mathrm{mg} / \mathrm{kg} \\
+ \text { vitamin } \\
\mathrm{C}\end{array}$ & $\begin{array}{c}\text { vitamin } \\
\mathrm{C} \& \\
\mathrm{MSG} \\
4 \mathrm{gm} / \mathrm{kg} \\
+ \\
\text { vitamin } \\
\mathrm{C}\end{array}$ \\
\hline 0.768 & $0.001 * *$ & $0.001 * *$ & 0.712 & 0.075 & $0.001 * *$ & $0.001 * *$ & 0.417 & 0.087 \\
\hline $\begin{array}{c}\mathrm{MSG} \\
100 \mathrm{mg} / \mathrm{kg} \& \\
\mathrm{MSG} \\
4 \mathrm{gm} / \mathrm{kg}\end{array}$ & $\begin{array}{c}\mathrm{MSG} \\
100 \mathrm{mg} / \mathrm{kg} \& \\
\mathrm{MSG} \\
100 \mathrm{mg} / \mathrm{kg} \\
+ \text { vitamin C }\end{array}$ & $\begin{array}{l}\mathrm{MSG} \\
4 \mathrm{gm} / \mathrm{kg} \& \\
\mathrm{MSG} \\
4 \mathrm{gm} / \mathrm{kg}+ \\
\text { vitamin C }\end{array}$ & $\begin{array}{l}\text { MSG } \\
100 \mathrm{mg} / \mathrm{kg}+ \\
\text { vitamin C\& } \\
\mathrm{MSG} 4 \mathrm{gm} / \mathrm{kg} \\
+ \text { vitamin C }\end{array}$ & & & & & \\
\hline $0.001 * *$ & $0.001 * *$ & $0.001 * *$ & $0.001 * *$ & & & & & \\
\hline
\end{tabular}

$N O=$ number; $* p<0.05$ significant $; * *<<0.001$ highly significant. 
Table (4): ANOVA one way statistical analysis of survivin expression among all studied groups.

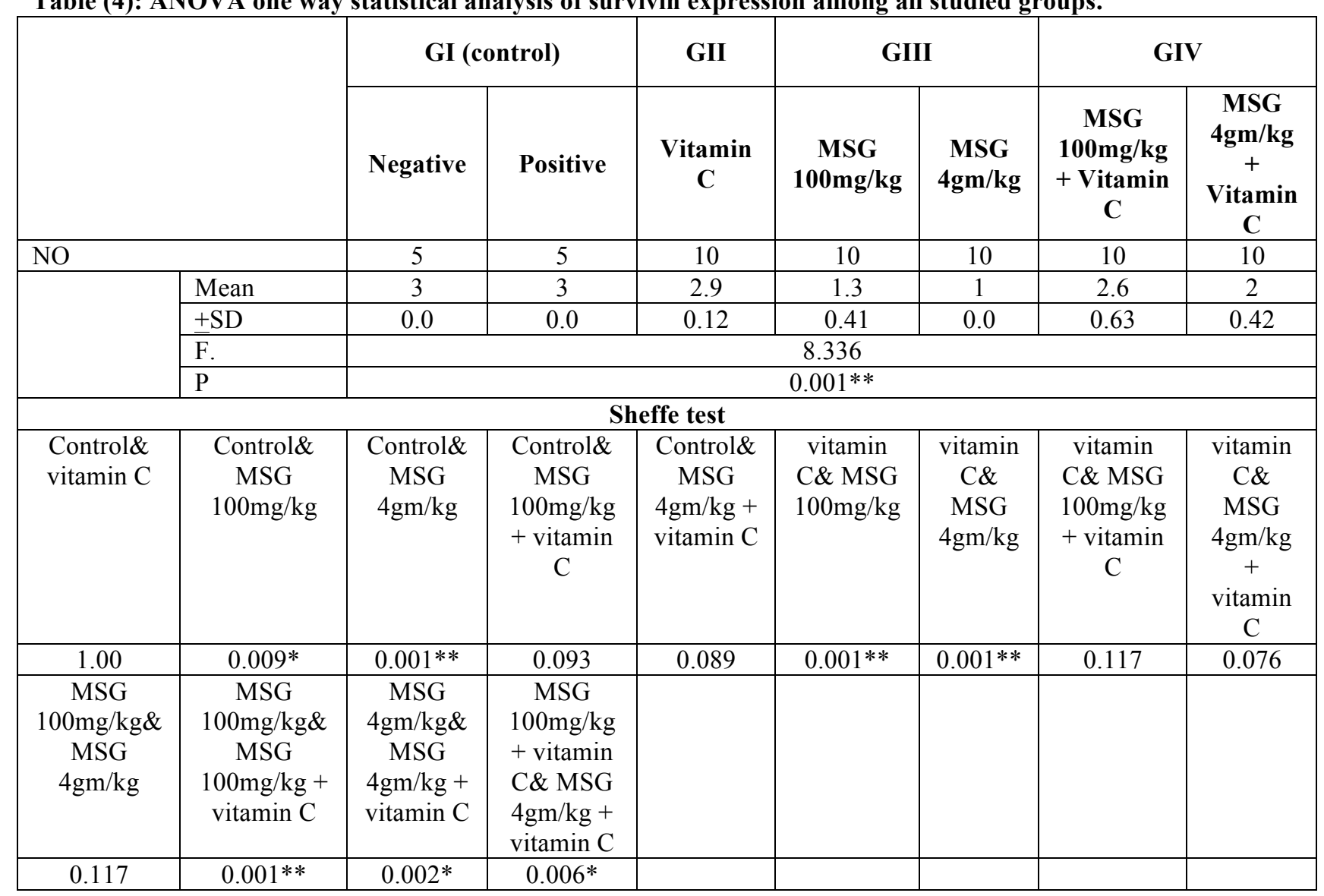

$N O=$ number $; * p<0.05$ significant $; * p<0.001$ highly significant.

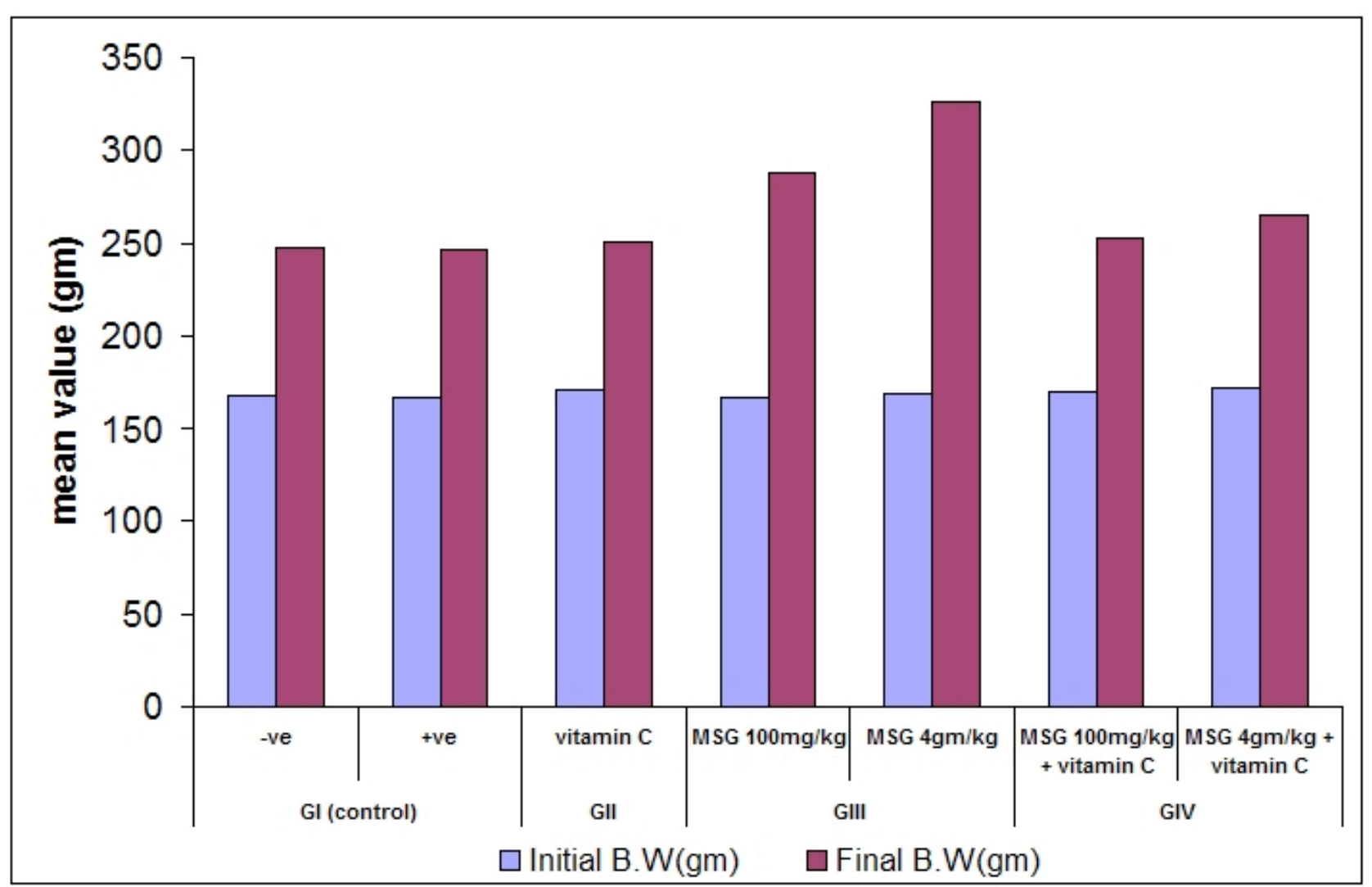

Figure (1): Mean values of the initial and final body weights (gm) of adult male rats of all studied groups (B.W= body weight). 


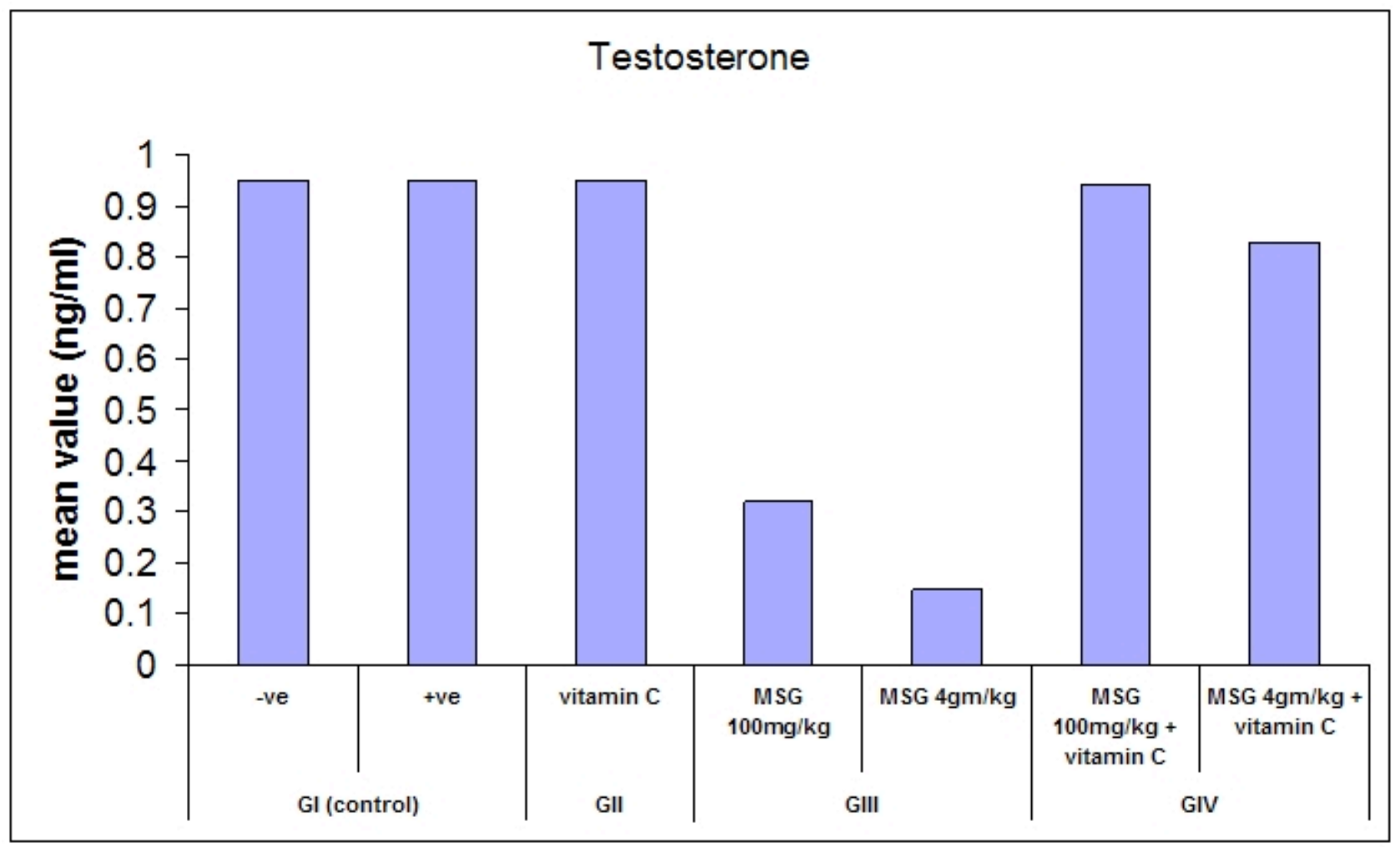

Figure (2): Mean values of serum testosterone level (ng/ml) in all studied groups.

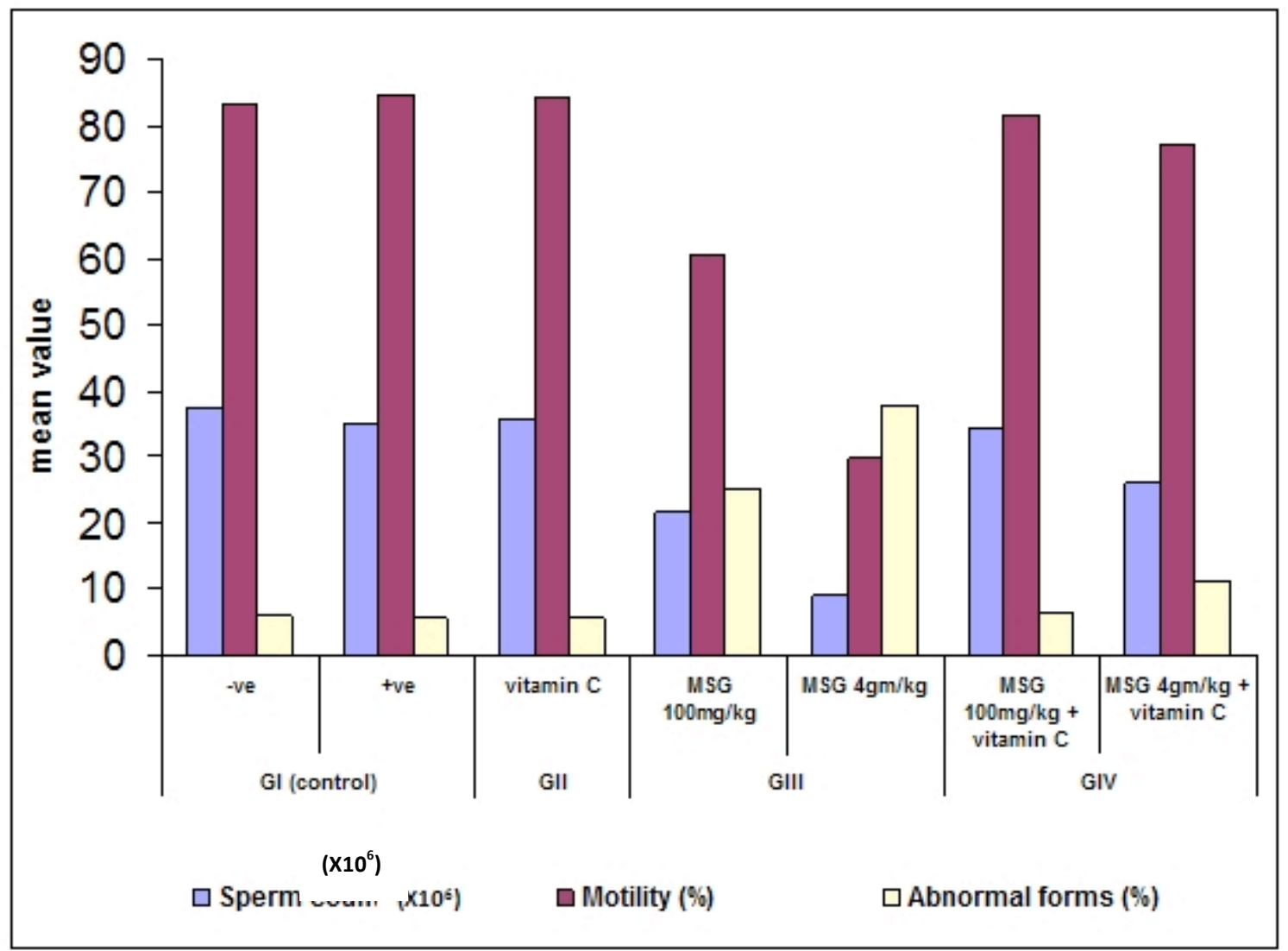

Figure (3): Mean values of cauda epididymal sperm reserves as regard sperm count (X10 ), sperm motility (\%) and incidence of abnormal forms (\%) among different studied groups. 


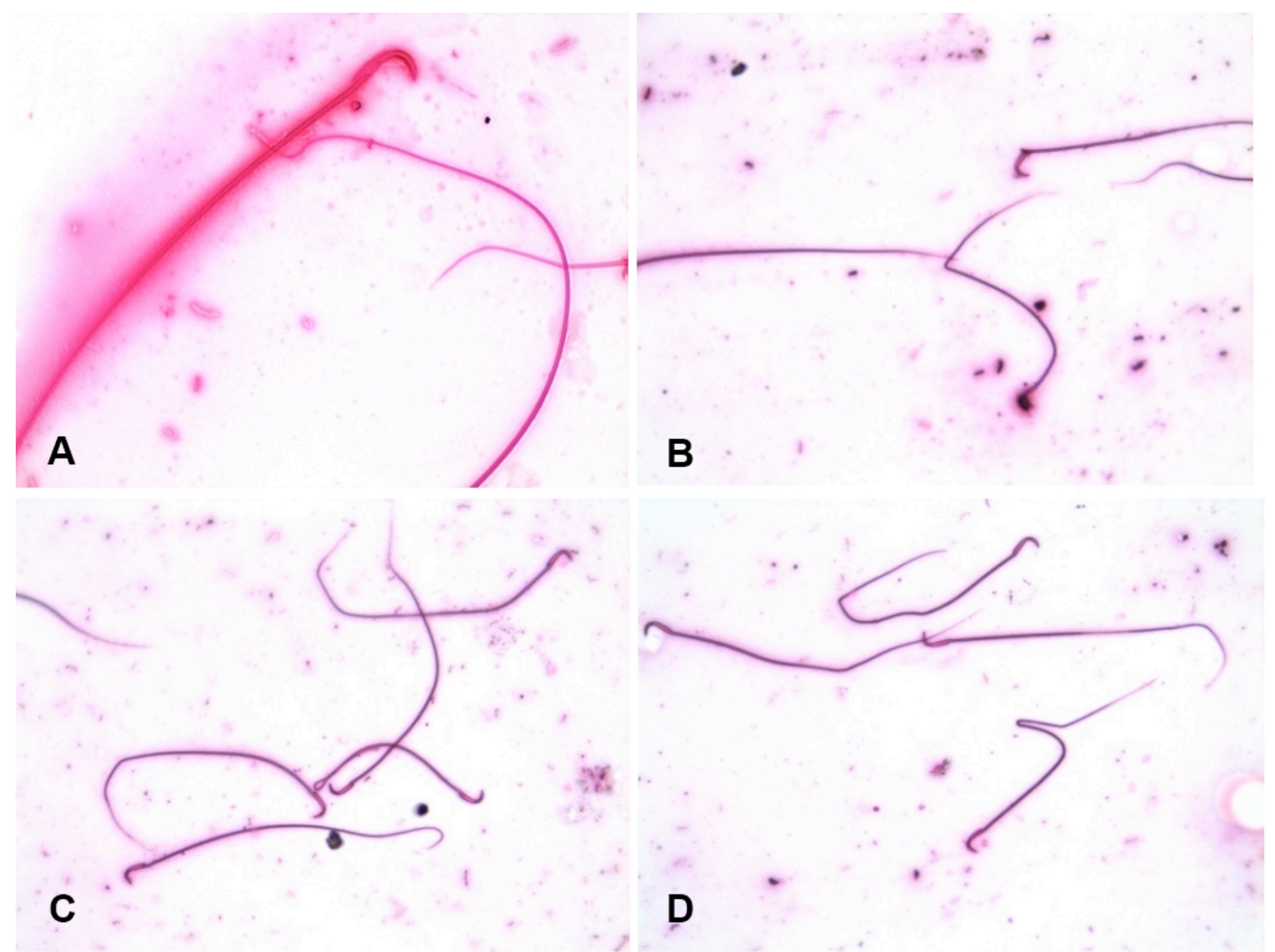

Figure (4): Photomicrograph showing normal and abnormal forms of sperms. A: normal sperm. B: sperm with bent neck. C: sperm with bent tail. D: sperm with bent neck and tail (H\&E x 400).

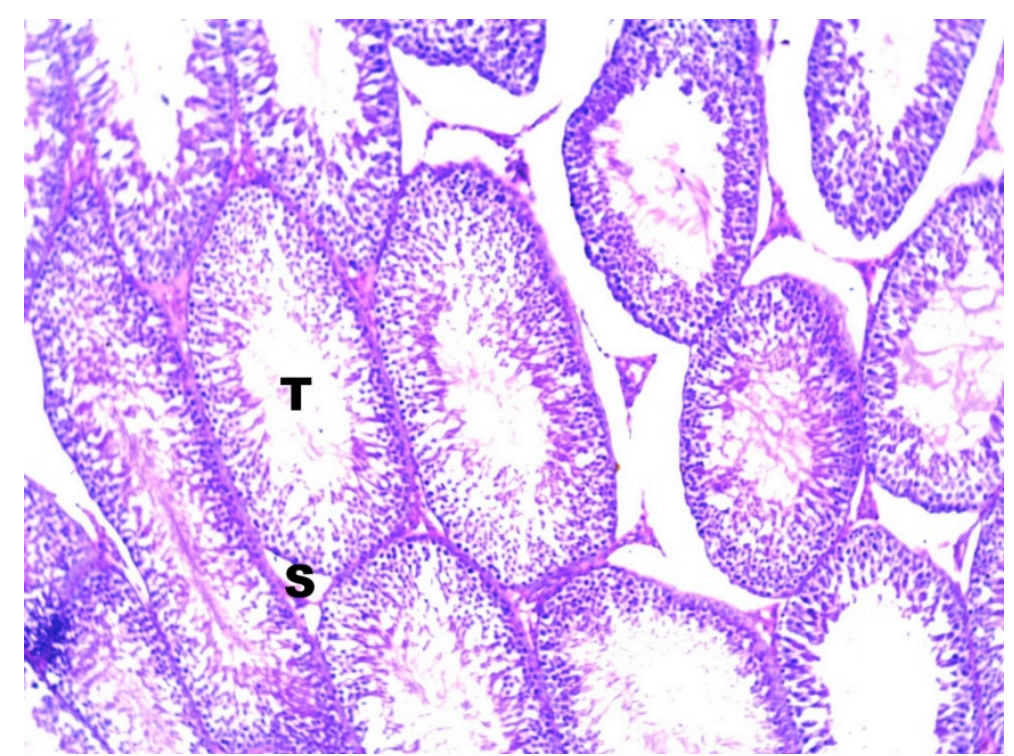

Figure(5): A photomicrograph of a cross section in a testis of an adult male albino rat of the control group (group I) showing normal testicular histology and normal spermatogenic progression. Note the active seminiferous tubules (T) and interstitial spaces (S). (H\&E stain X200). 


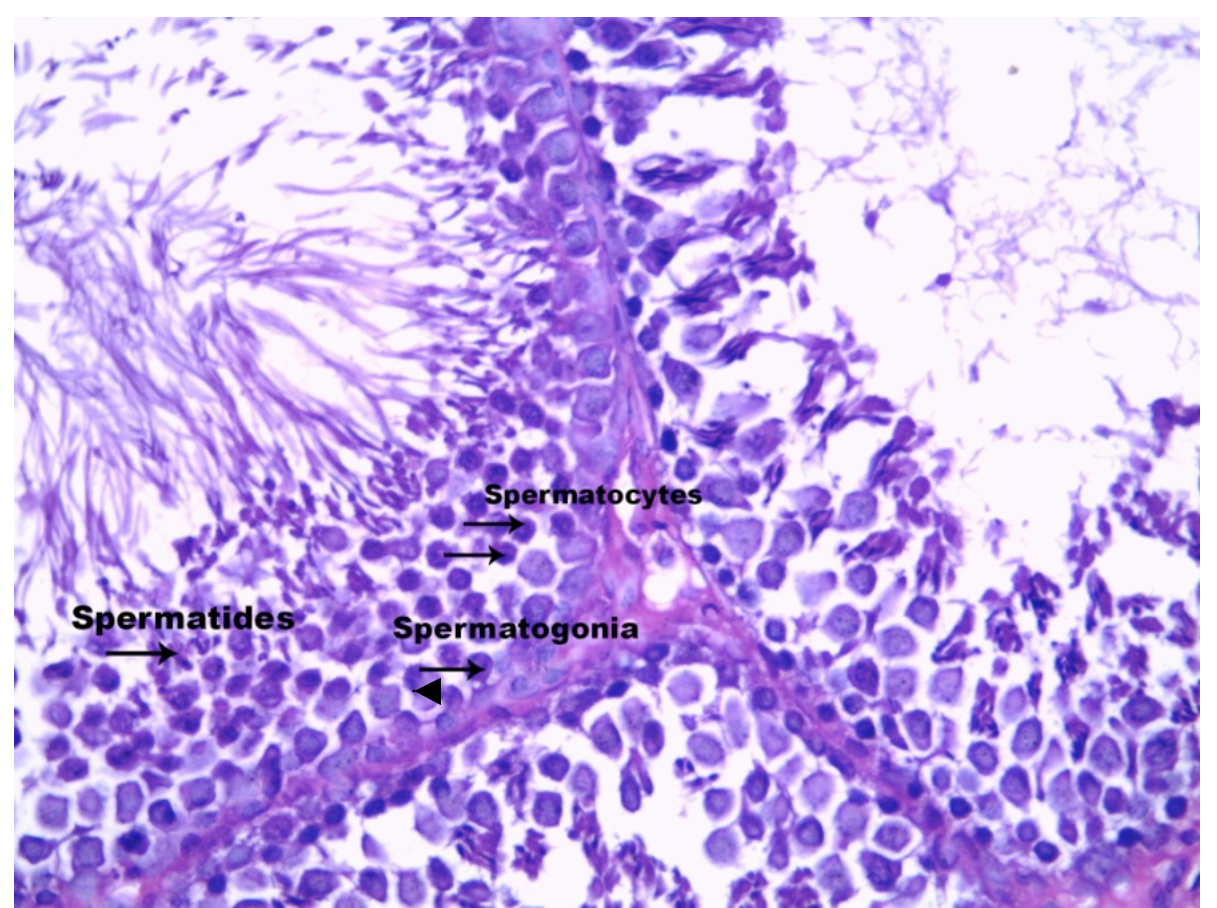

Figure (6): A photomicrograph of a section in the seminiferous tubule of an adult male albino rat of the vitamin C treated group (group II) showing unremarkable histopathological changes. The seminiferous epithelium shows active spermatogenesis. (H\&E stain X400).

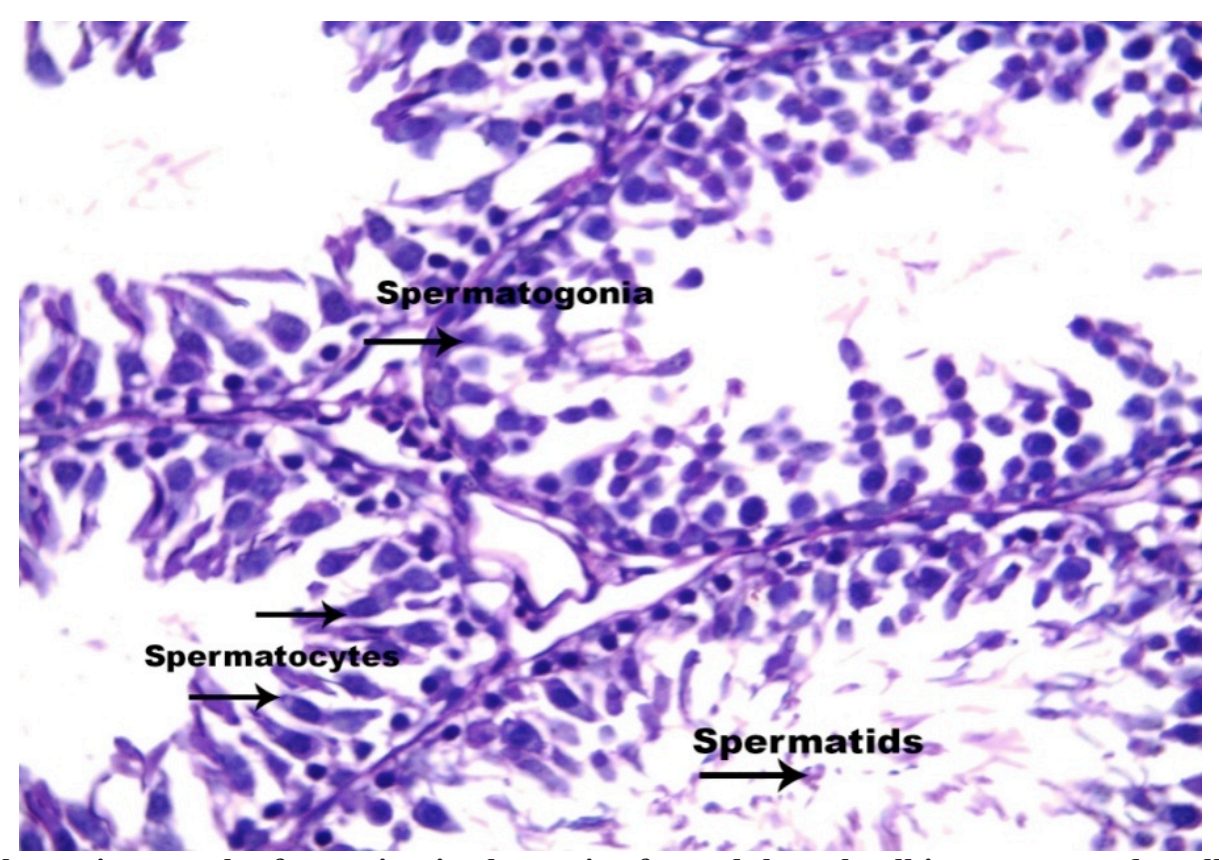

Figure (7): A photomicrograph of a section in the testis of an adult male albino rat treated orally by MSG in a dosage of $100 \mathrm{mg} / \mathrm{kg}$ showing damage of spermatogonia and elongation of primary spermatocytes. Few damaged late spermatids are still present in the tubules. (H\&E stain X400). 


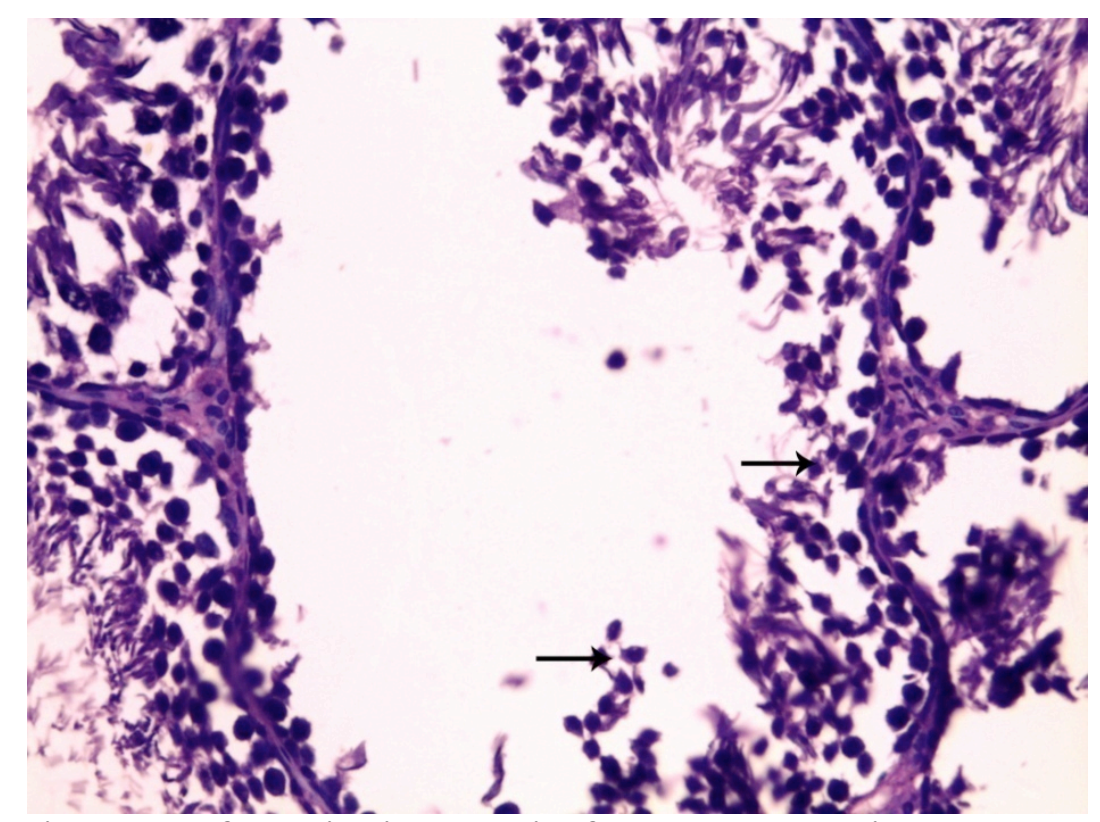

Figure (8): A photomicrograph of a section in the testis of an adult male albino rat treated orally by MSG in a dosage of $4 \mathrm{gm} / \mathrm{kg}$ showing a severely damaged seminiferous tubule. Few primary spermatocytes (arrows)are still seen in the tubule(H\&E stain X400).

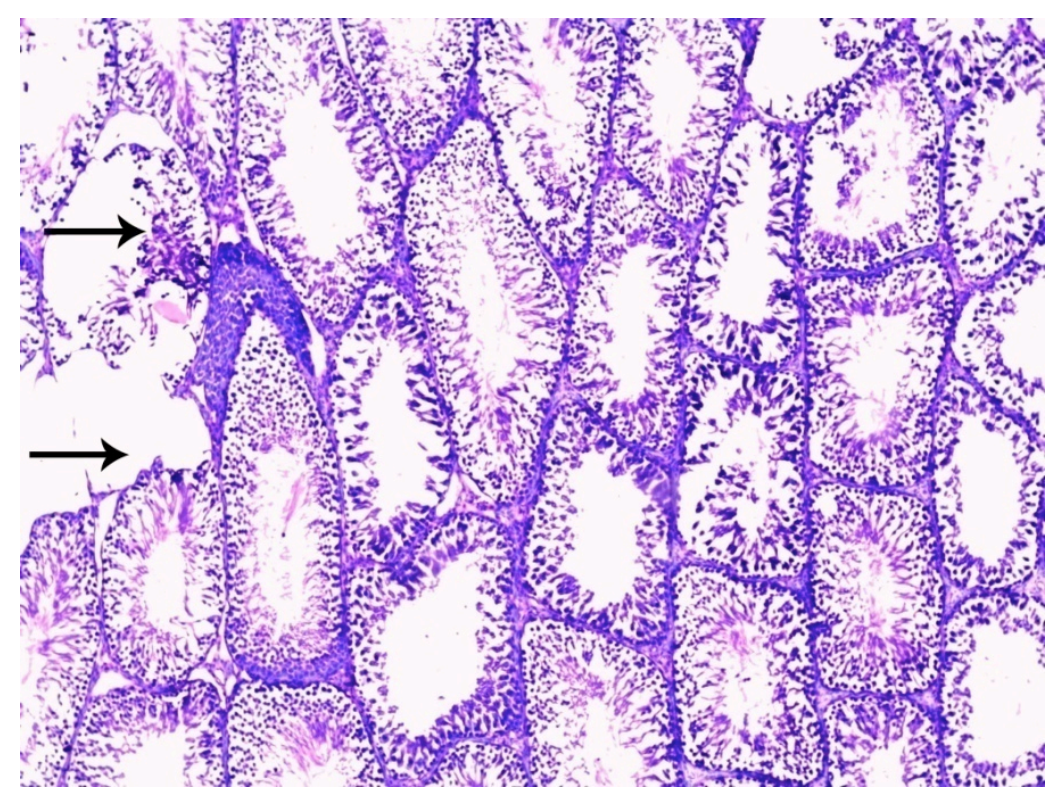

Figure (9): A photomicrograph of a section in the testis of an adult male albino rat treated orally by $100 \mathrm{mg} / \mathrm{kg}$ MSG and $100 \mathrm{mg} / \mathrm{kg}$ vitamin $\mathrm{C}$ showing some normal seminiferous tubules with normal spermatogensis admixed with few degenerated tubules (arrows) (H\&E stain X200). 


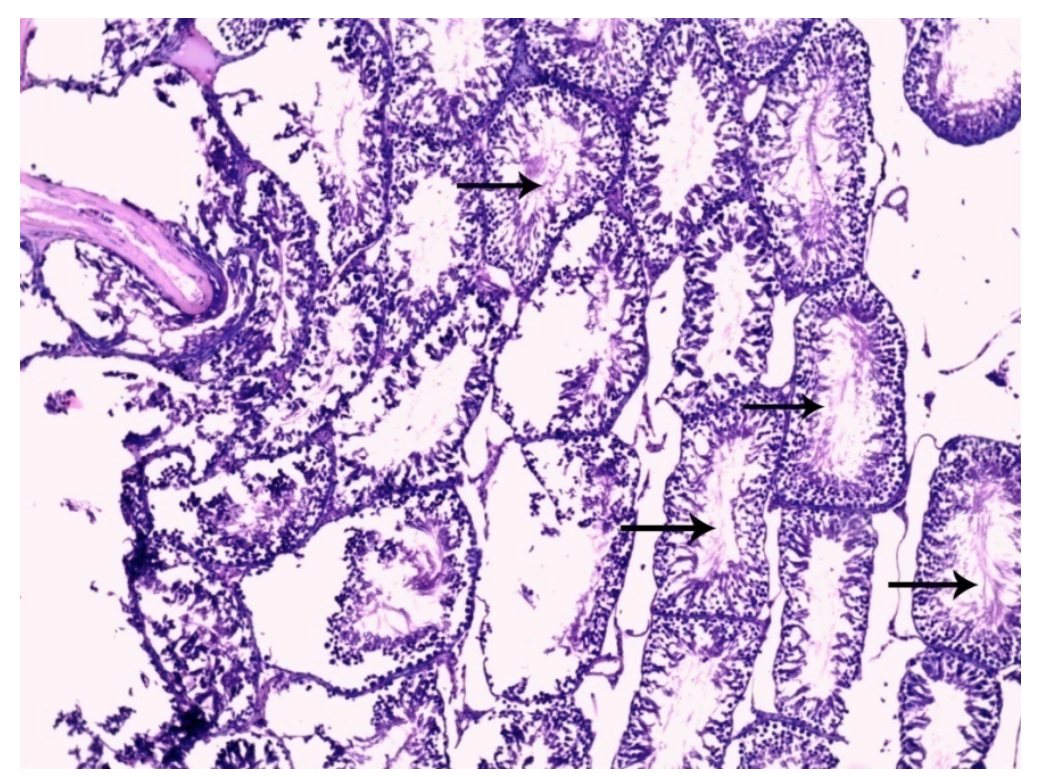

Figure (10): A photomicrograph of a cross-section of seminiferous tubules of a testis of an adult male albino rat treated orally by $4 \mathrm{gm} / \mathrm{kg}$ MSG and $100 \mathrm{mg} / \mathrm{kg}$ vitamin $\mathrm{C}$ showing few preserved normal seminiferous tubules with normal spermatogensis (arrows) admixed with severely damaged tubules (H\&E stain X200).

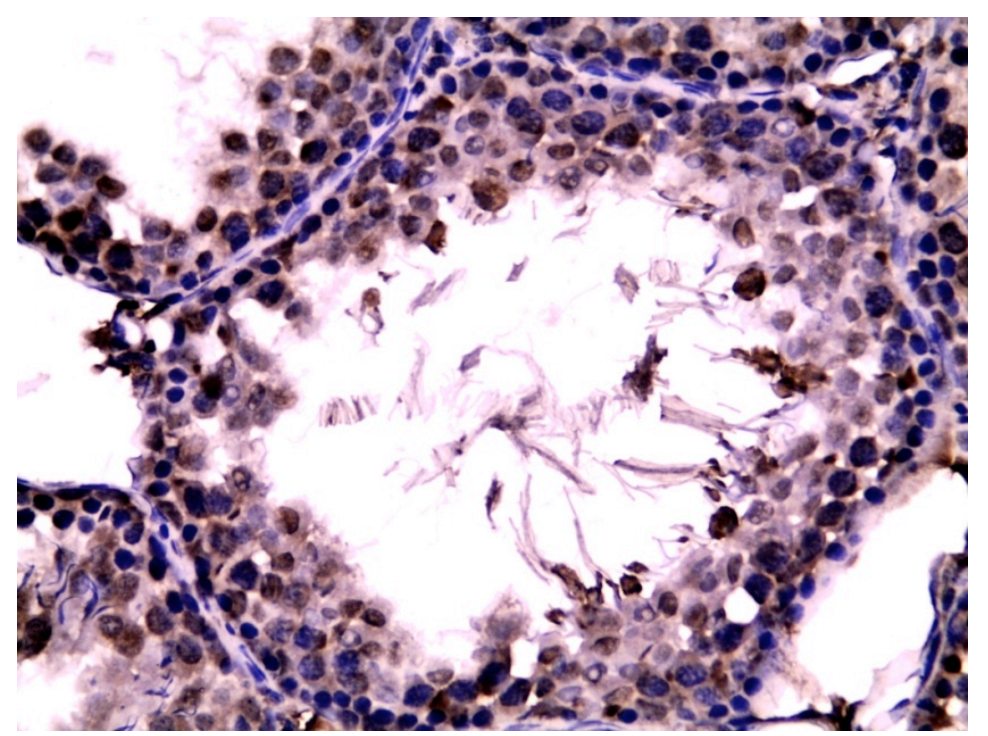

Figure (11): A photomicrograph of a section in the seminiferous tubule of an adult male albino rat of control and vitamin treated rats (group II) shows strong survivin immunostaining in most of the nuclei and cytoplasm of spermatogenic cells (IHCx400). 


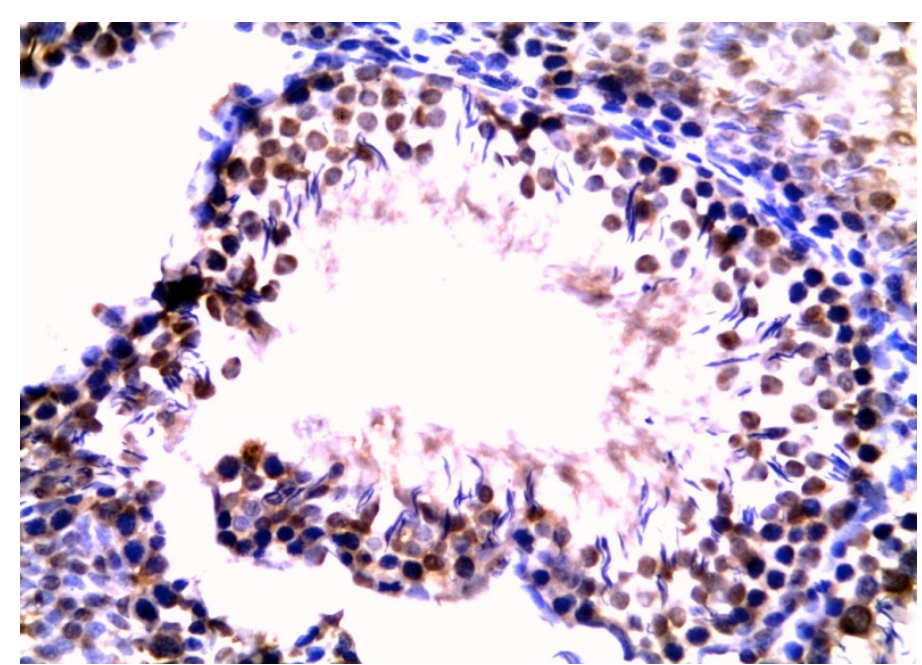

Figure (12): A photomicrograph of a section in the seminiferous tubule of an adult male albino rat treated orally by MSG in a dosage of $100 \mathrm{mg} / \mathrm{kg}$ rat showing mild nuclear and cytoplasmic survivin immunostaining of the spermatogenic cells (IHCx400).

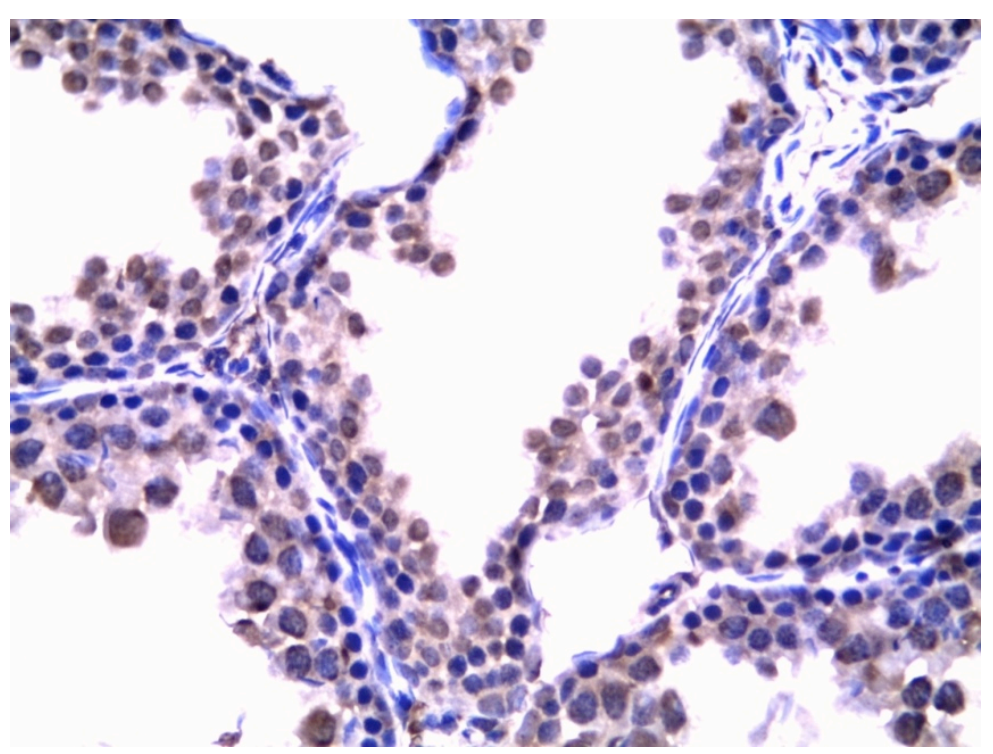

Figure (13): A photomicrograph of an adult male albino rat treated orally by MSG in a dosage of $4 \mathrm{gm} / \mathrm{kg}$ (IHCx400) showing marked reduction in survivin expression in the nuclei and cytoplasm of spermatogenic cells of most seminiferous tubules.

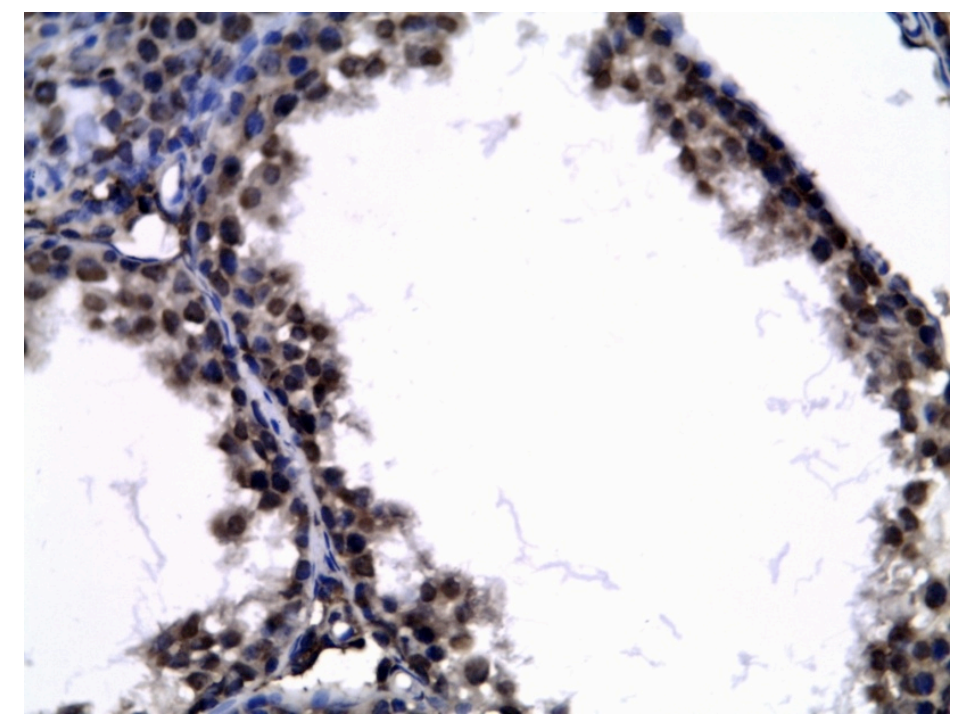

Figure (14): A photomicrograph of a section of seminiferous tubule of an adult male albino rat treated orally by $100 \mathrm{mg} / \mathrm{kg}$ MSG and $100 \mathrm{mg} / \mathrm{kg}$ vitamin $\mathrm{C}$ showing strong nuclear and cytoplasmic surviving immunostaining in the spermatogenic cells (IHCx400). 


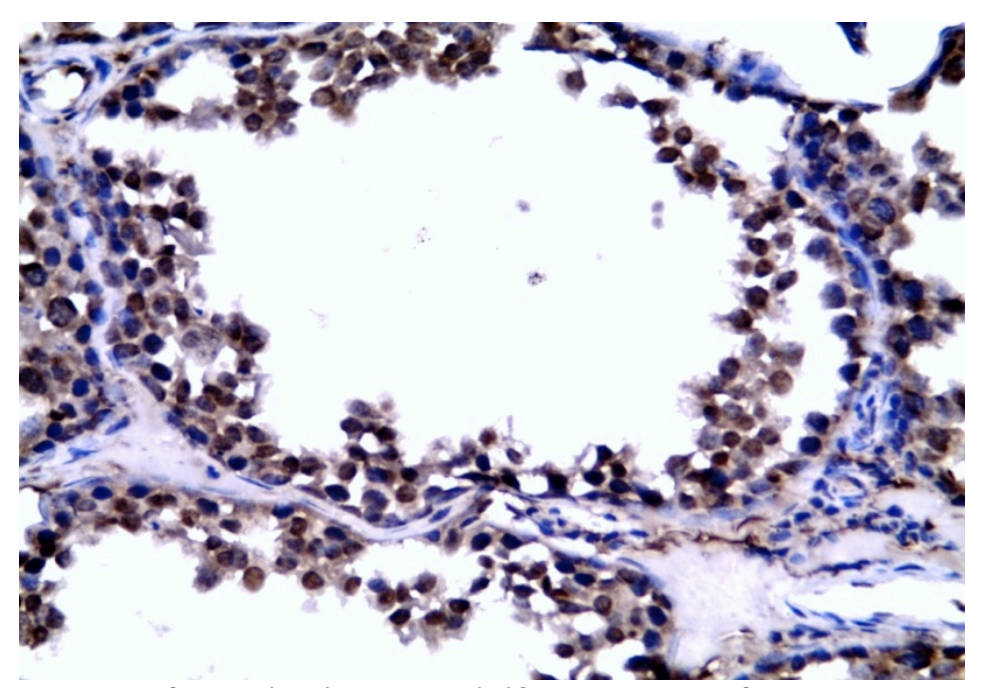

Figure (15): A photomicrograph of a section in the seminiferous tubule of an adult male albino rat treated orally by $4 \mathrm{gm} / \mathrm{kg}$ MSG and $100 \mathrm{mg} / \mathrm{kg}$ vitamin $\mathrm{C}$ showing moderate nuclear and cytoplasmic surviving immunostaining in spermatogenic cells. (IHCX400).

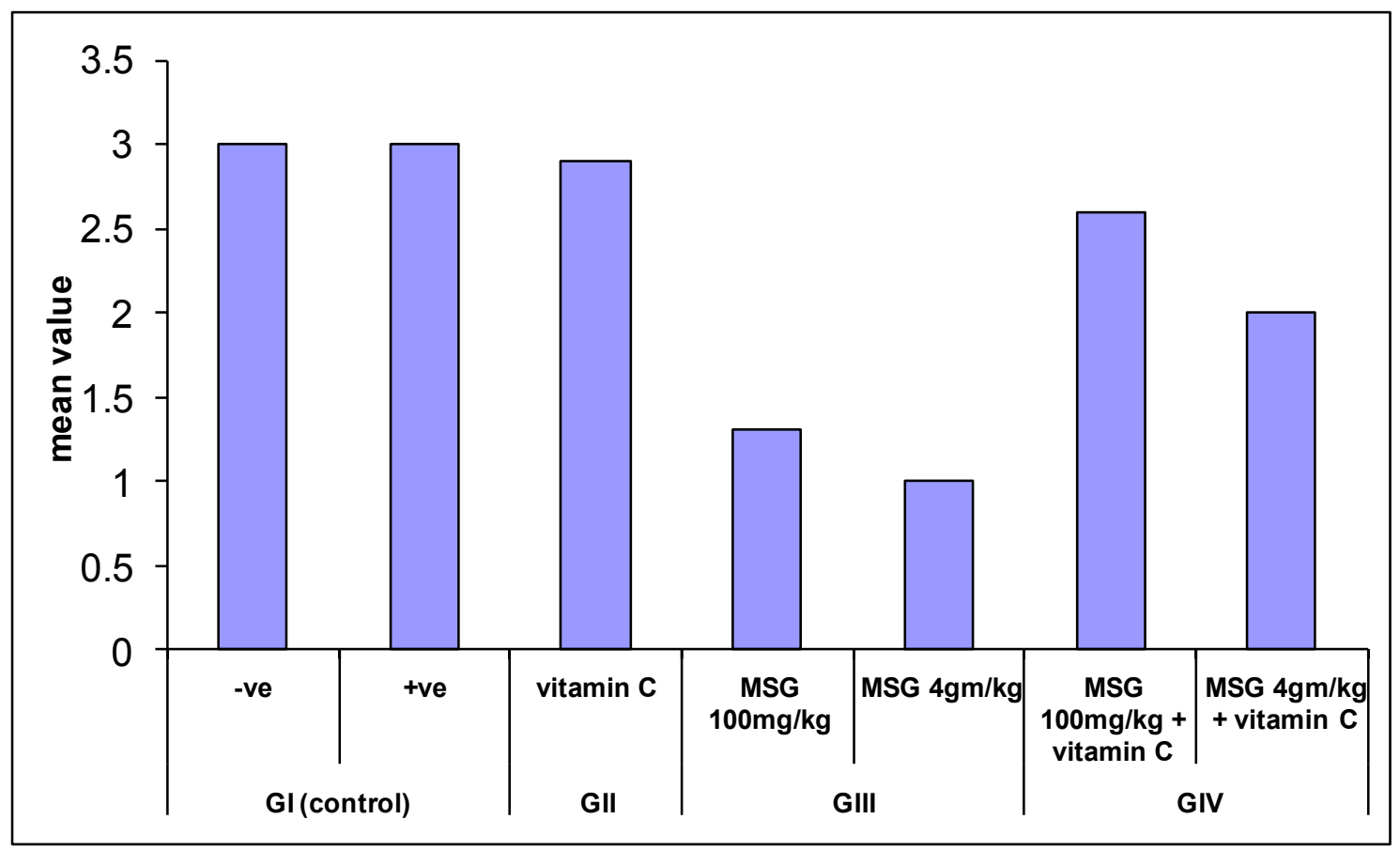

Figure (16): Mean values of survivin expression in testes sections among all studied groups.

\section{Discussion}

Glutamate was reported to have many functions in the human body as a metabolic intermediate and protein constituent (Zai et al., 2009). Despite its taste stimulation and improved appetite enhancement, reports indicate that $\mathrm{MSG}$ is toxic to human and experimental animals (Belluardo et al., 1990). MSG leads to generation of free radicals, activation of proteases, phospholipases and endonucleases, transcriptional activation of apoptotic programs and genotoxicity in mouse and rat (Goldsmith, 2000 and Farombi and Onyema, 2006).

MSG has high excitotoxic potential, which is mediated at the level of second messenger by calcium anions (Gao et al., 1994 and Lin et al., 1995). Bojanic et al., (2009) hypothesized that high concentration of
MSG could alter permeability of neural membrane for calcium which could be involved in the mechanisms of MSG toxicity. Calcium ion influx into cells accompanies cell injury caused by a variety of stimuli, including oxidative stress and calcium also appears to be involved in the production of reactive oxygen species (ROS). Hydrogen peroxide (which can produce ROS) induces raised intracellular calcium levels, the response being suppressed by antioxidants (Feng and Hart, 1996).Being rich in membrane bound polyunsaturated fatty acids (PUFA), mammalian spermatozoa are more susceptible to ROS attack (Lenzi et al., 2000).

In the present study, toxicity of MSG in low dose $(100 \mathrm{mg} / \mathrm{kg})$ and high dose $(4 \mathrm{gm} / \mathrm{kg})$ was 
evaluated on adult male albino rat testis and epididymis and possible ameliorative effect of vitamin $\mathrm{C}$. The rat model is probably the best choice in the experimental study because purchase and harvesting are inexpensive. In addition, spermatogenesis observed in the rat is similar to that in humans (Turner, 2001).

There was a significant increase in the body weight of rats treated with MSG compared with control or vitamin $\mathrm{C}$ treated animals. Moreover, there was more significant increase in the body weight in $4 \mathrm{gm} / \mathrm{kg}$ than in $100 \mathrm{mg} / \mathrm{kg}$ MSG- treated animals.

These results were in agreement with Bloom, (2001) who suggested that MSG affected hypothalamus which is responsible for appetite, body temperature, water balance and sleep. These results are also in line with the study carried out by Nakagawa et al. (2000) and Ortiz et al. (2006).

Other studies have also shown that (MSG) causes reduction in the secretion of growth hormones, leading to stunted growth and irreversible obesity, essentially due to the accumulation of excess fats in adipose tissue (Ikonomidou et al., 1995; Eskes, 1998 and Rodriquez et al., 1998). Through its stimulation of the orosensory receptors and by improving the palatability of meals, MSG influences the appetite positively, and induces weight gain (Rogers and Blundell, 1990). Macho et al. (2000) had cited that obesity induced by MSG was due to chemical lesions in arcuate nucleus structure that resulted in endocrine disorders such as obesity and glucose intolerance.

The current work demonstrated a significant decrease in the level of serum testosterone hormone in MSG treated groups compared to the control group and vitamin $\mathrm{C}$ treated group. This decrease in serum testosterone level was dose dependent. The testosterone level in $4 \mathrm{gm} / \mathrm{kg}$ MSG-treated animals was significantly reduced than in $100 \mathrm{mg} / \mathrm{kg}$. This was in agreement with Igwebuike et al., (2011) who reported marked reduction of serum testosterone level in both young and adult rats treated with MSG.

Neuronal losses in the hypothalamus can result in disruption of the hypothalamic-pituitary-testis regulatory axis that controls the steroidogenesis of testicular Leydig cells (McLachlan et al., 1996). This will ultimately impact serum testosterone levels, because $95 \%$ of the androgen is produced by Leydig cells of the testes, while cells in the cortex of the adrenal gland produce about 5\% (Urban, 1999). Such alterations in reproductive hormone secretion may adversely affect the reproductive capacity of the affected animals.

Because testosterone plays a crucial role in male reproductive system, disruption of its production may impair male reproductive health (McLachlan et al., 2002 and O'Shaughnessy et al., 2010). Testosterone is needed for the continued production of different generation of germ cells in the seminiferous tubules. Therefore, reduction of testosterone level may lead to the separation of germ cells from the epithelium of the seminiferous tubules, decreased daily sperm production and morphological abnormality of testis (Blanco-Rodriguez and Martinez-Garcia, 1998 and Zitzmann, 2008). This was evidenced in the present study by histological examination of testis treated with MSG where there was damage of spermatogonia and elongation of primary spermatocytes with few damaged late spermatids in the tubules in $100 \mathrm{mg} / \mathrm{kg}$ MSG-treated animals. While in $4 \mathrm{gm} / \mathrm{kg}$ MSG- treated group there was severely damaged seminiferous tubules with few primary spermatocytes in the tubules.

Similarly, Eweka and Om'iniabohs, (2011)reported that male rats treated with $6 \mathrm{gm}$ of MSG showed cystic degenerative changes, with some intercellular vacuolations appearing in the stroma of the seminiferous tubules when compared to the control sections. Also, Mohamed, (2012) observed structural changes of testes of MSG-treated rats which were dosage-duration-dependent. These changes ranged from slight to moderate damage in case of the shortterm treatment however, severe damage of spermatogenic and Sertoli cells was recorded in case of the long-term treatment.

This was in contrast with Igwebuike et al. (2011) who reported the absence of any overt pathological lesions in the histology of the testes of the young and adult rats that received varied doses of monosodium glutamate suggests that MSG did not have any direct toxic effect on the testes.

The present study revealed dose-dependent reduction in cauda epididymal sperm counts in the MSG-treated animals compared to control and vitamin $\mathrm{C}$ treated groups. Reduction in sperm count was more significant in $4 \mathrm{gm} / \mathrm{kg}$ treated animals. This was in agreement with Igwebuike et al., (2011). The dosedependent reduction in cauda epididymal sperm counts following oral administration of monosodium glutamate to both young and adult male rats may be an indication that the normal process of spermatogenesis was adversely affected in the rats that consumed monosodium glutamate. Spermatogenesis is a complex cyclic process in which germ cells undergo series of mitotic and meiotic cell divisions, followed by morphological differentiation in a delicately regulated spatiotemporal fashion in the seminiferous epithelium. In mammals, spermatogenesis is totally dependent upon testosterone (Pakarainen et al., 2005 and Wang et al., 2009); however, only total abolition of testicular androgen action resulted in consistent azoospermia (Zhang et al., 2003).

As regards, androgens act to stimulate spermatogenesis through androgen receptors (ARs) located on Sertoli cells and peritubular myoid cells of the testis (De Gendt et al., 2004). It has been demonstrated that androgen stimulation of spermatogenesis requires direct androgen action on the Sertoli cells (O'shaughnessy et al., 2010), and this was necessary for postmeiotic spermiogenesis (Zhang et al., 2003). Thus, appropriate regulation of androgen activity via the hypothalamic-pituitary-testis axis is necessary for initiation and maintenance of spermatogenesis. The indication is that the reduced cauda epididymal sperm counts observed in the MSGtreated rats in the present study may be the end result of a considerable decline in the influence of testosterone on spermatogenesis in these rats. 
Furthermore, there was a significant increase in the abnormal sperm morphology and a significant decrease in sperm motility in MSG treated groups compared to control and vitamin $\mathrm{C}$ treated groups. This effect was more noticed in $4 \mathrm{gm} / \mathrm{kg}$ MSG- treated animals than in $100 \mathrm{mg} / \mathrm{kg}$ MSG- treated animals.

The toxic effect of MSG on the testes was reported by other studies where a significant oligozoospermia and increase abnormal sperm morphology in a dose-dependent fashion in male Wistar rats were observed (Onakewhor et al., 1998).Also, it has been established that MSG may be implicated in cases of male infertility as it causes testicular hemorrhage, degeneration and alteration of sperm cell population and morphology (Oforofuo et al., 1997).

The primary mechanism for glutamate induced cell death is apoptosis in different cell types (Martin et al., 2000 and Schelman et al., 2004).Apoptosis of selected germ cells occurs normally in the testis and is essential for the normal maintenance of spermatogenesis (Haouzi et al., 2008). Among the mammalian apoptosis regulators, the inhibitor of the apoptosis (IAP) protein family is just beginning to be elucidated for its role in spermatogenic function.

Survivin is an IAP protein regulating apoptosis at cell division. Survivin has been studied extensively in cancer cells, but has only recently been studied extensively in male germ cells. In the rodent testis, survivin expression has been localized to germ cells, especially mature spermatocytes. Testicular survivin was found to be one of the highly expressed transcripts in adult human testis, specially in the nuclei of mature spermatocytes, and its down-regulation is associated with spermatogenic failure (Weikert et al., 2004; Weikert et al., 2006; Absalan et al.,2010 and AlMaghrebi et al., 2011).The fact that survivin regulates cell proliferation, cell cycle, and programmed cell death makes it a candidate gene that may be involved in the regulation of germ cell production (Fukuda et al., 2002).

Survivin expression in the cytoplasm could be associated with its function in controlling cell survival (inhibitor of apoptosis), whereas nuclear staining may rather promote cell proliferation (Taubert et al., 2010). As an inhibitor of apoptosis, survivin is believed to block apoptosis by inhibiting caspases and antagonizing mitochondria-dependent apoptosis (Marusawa et al., 2003 and Sah et al., 2006). In contrast, survivin activity is blocked through its interaction with the mitochondrial protein; S-mac, which is released into the cytoplasm as a response to apoptotic stimuli, rendering survivin unable to inhibit caspases (Ceballos-Cancino et al., 2007). This finding suggested that suppression of survivin expression could promote the apoptotic stimuli. The potential mechanism of survivin down-regulation has been suggested by several studies to be secondary to ROS generation and ROS-induced apoptosis (Seo et al., 2007). Also, McCrann et al. (2009) have shown that over-expression of Nox4, a member of the NADPH oxidase family and a major source for ROS production has resulted in down-regulation of survivin mRNA. These findings led to the hypothesis that one of the manifestations of deregulated ROS signaling could be the down-regulation of survival genes.

In the present study, there was a significant down-regulation of survivin in MSG-treated animals compared to control and vitamin $\mathrm{C}$ treated groups. Reduction in survivin expression in MSG-treated rats was dose dependent. It was more marked in animals treated with $4 \mathrm{gm} / \mathrm{kg}$ that indicated more liability for cell death with higher dose.

Vitamin C (ascorbic acid) has a well-known antioxidant protective role as it is considered the front line of defense against free radicals through; reactive oxygen species scavenging, reduction of peroxides and repair of peroxidized biological membranes and sequestration of iron (Loo et al. 2003).

In the male reproductive system, vitamin $\mathrm{C}$ is known to protect spermatogenesis.It increases gamete mobility since it reduces oxidative stress level (Hsu et al., 1998). Also, it plays a major role in semen integrity and fertility both in men and animals (Agarwal et al., 2005 and Eskenazi et al., 2005), increases testosterone levels (Sönmez et al., 2005) and prevents sperm agglutination. Moreover, it is an important chainbreaking antioxidant, contributing up to $65 \%$ of the total antioxidant capacity of seminal plasma found intracellulary and extracellulary (Makker et al., 2009).

In the present study, protective effect of vitamin C was observed in MSG- vitamin C treated groups. There was significant reduction in the body weight in MSG-vitamin C treated groups compared to MSG -treated groups which was more significant in $100 \mathrm{mg} / \mathrm{kg}$ MSG-vitamin $\mathrm{C}$ than in $4 \mathrm{gm} / \mathrm{kg}$ MSGvitamin $\mathrm{C}$ treated animals.

Also, there was a significant improvement in serum testosterone levels, sperm count and sperm motility with decreased abnormal forms in MSGvitamin $\mathrm{C}$ treated animals compared to MSG-treated animals. These effects were more significant in $100 \mathrm{mg} / \mathrm{kg}$ MSG treated animals than in $4 \mathrm{gm} / \mathrm{kg} \mathrm{MSG}$ treated ones.

Histological examination has confirmed this protective effect of vitamin $\mathrm{C}$ on MSG -treated animals. This protection was more marked in animals treated with $100 \mathrm{mg} / \mathrm{kg}$ MSG where some normal seminiferous tubules with normal spermatogensis admixed with few degenerated tubules were observed, while in $4 \mathrm{gm} / \mathrm{kg}$ MSG -vitamin C treated animals showed few preserved normal seminiferous tubules with normal spermatogensis admixed with severely damaged tubules. As regards survivin expression, there was strong nuclear and cytoplasmic expression in the spermatogenic cells in animals given vitamin $\mathrm{C}$ with MSG in a dosage of $100 \mathrm{mg} / \mathrm{kg}$ while moderate nuclear and cytoplasmic immuno-staining of spermatogenic cell was observed in animals given vitamin $\mathrm{C}$ with MSG in a dosage of $4 \mathrm{gm} / \mathrm{kg}$.

These findings were in consistence with Farombi and Onyema, (2006) who suggested that antioxidants such as vitamin $\mathrm{C}$ have protective potential against oxidative stress induced by MSG. Furthermore, Shrilatha and Muralidhara, (2007) have 
reported the protective effect of vitamin $\mathrm{C}$ on testicular oxidative stress, sperm oxidative stress and genotoxic effects using a diabetic mice model. Similarly, Naziroğlu (2003) concluded that vitamin C acted as antioxidant in reproductive milieu. Also, Duarte and Lunec, (2005) and Li and Schellhorn, (2007) have reported that vitamin $\mathrm{C}$ attenuated the reduction of seminiferous tubular diameters probably because this vitamin may have a role in the synthesis of extracellular matrix components.

\section{Conclusions}

The present work has demonstrated that oral administration of monosodium glutamate resulted in lowered serum testosterone levels and reduction in the cauda epididymal sperm reserves of adult male rats and cause overt pathological lesions in their testes. These effects were dose dependent and oral therapeutic administration of vitamin $\mathrm{C}$ along with MSG provided a significant improvement in the biochemical and pathological changes in testes of rats administrated MSG only at low dose $(100 \mathrm{mg} / \mathrm{kg})$ in comparison with high dose $(4 \mathrm{gm} / \mathrm{kg}) \mathrm{MSG}$ treated group. Thus, vitamin $\mathrm{C}$ holds a promise as an agent that can potentially reduce MSG induced toxic effects in testes.

\section{Recommendations}

Extra-antioxidant supplementation through diets can be helpful as a defense mechanism and might be helpful in prevention of oxidative stress and allied aliments. Further studies on animals and/or human are recommended to study the protective and preventive effects of different anti-oxidants, also to know if this toxic effect is reversible or not.

\section{References}

Absalan F, Movahedin M and Mowla SJ (2010): Germ cell apoptosis induced by experimental cryptorchidism is mediated by molecular pathways in mouse testis. Andrologia 42: 512.

Agarwal A, Prabakaran SA and Said TM (2005): Prevention of oxidative stress injury to sperm. J Androl 26(6):654-660.

Al-Maghrebi M, Kehinde EO and Anim JT (2011):Survivin downregulation is associated with vasectomy-induced spermatogenic damage and apoptosis. Med PrincPract 20: 449-454.

Bancroft JD and Gamble M (2002): Theory and practice of histological techniques, $5^{\text {th }}$ edition, Churchll Livingstone, Edinburgh, London, New York, Tokyo, P.139

Bearden HJ and Fuquay JW (1997): Semen evaluation. In: Applied Animal Reproduction. Stewart, C.E. (Ed.), $4^{\text {th }}$ ed. Prentice Hall, Inc., United States of America, Mexico and Tokyo, pp. 158-169.

Bellisle F (2008): Experimental studies of food choices and palatability responses in European subjects exposed to the Umami taste. Asia Pac J Clin Nutr 17(S1): 376-379.

Belluardo M, Mudo G and Bindoni M (1990): Effect of early Destruction of the mouse arcuate nucleus by MSG on age Dependent natural killer activity. Brain Res 534:225-333

Blanco-Rodriguez J and Martinez-Garcia C (1998): Apoptosis precedes detachment of germ cells from the seminiferous epithelium after hormone suppression by short-term oestradiol treatment of rats. Int J Androl 21: 109-15.

Bloom FE (2001): Neurotransmission and the Central Nervous System. In: The Pharmacological Basis of Therapeutics, 9th Edn, Hardmann GL, Limblrd LE, Molinoff PB, Ruddon RW and Gilman AG (Eds.). McGraw-Hill, New York, pp: 267-290.

Bojanić V, Bojanić Z, Najman S, et al., (2009): Diltiazem prevention of toxic effects of monosodium glutamate on ovaries in rat. Gen PhysiolBiophys 28:149-54.

Ceballos-Cancino G, Espinosa M, Maldonado V, et al., (2007): Regulation of mitochondrial Smac/DIABLO-selective release by survivin. Oncogene 26:7569-7575.

De-Gendt K, Swinnen JV, Saunders PT, et al., (2004): A Sertolicellselective knockout of the androgen receptor causes spermatogenic arrest in meiosis. Proc. Natl. Acad. Sci. USA 101: 1327-1332.

Diniz YS, Fernandes AA, Campos KE, et al., (2004): Toxicity of hypercaloric diet and monosodium glutamate: oxidative stress and metabolic shifting in hepatic tissue. Food-and-Chemical Toxicology 42(2): 313-9.

Duarte TL and Lunec J (2005): When is an antioxidant not an antioxidant? A review of novel actions and reactions of vitamin C. Free Radic Res 39(7):671-686.

Egbuonu ACC and Osakwe ON (2011): Effects of high monosodium glutamate on some serum markers of lipid status in male Wistar rats. J Med \& Med Sci 2(1): 653-656.

Egbuonu ACC, Obidoa O, Ezeokonkwo CA, et al., (2009): Hepatotoxic effects of low dose oral administration of monosodium glutamate in male albino rats. African J. Biotechnol 8: 3031-3035.

Egbuonu ACC, Obidoa O, Ezeokonkwo CA, et al., (2010): Some biochemical effects of sub-acute oral administration of L-arginine on monosodium glutamate-fed Wistar albino rats 1: Body weight changes, serum cholesterol, creatinine, and sodium ion concentrations. Toxicol and Environ Chem 92(7):13311337.

Ehlers I, Niggemann B, Binder C, et al., (1998): Role of non allergic hypersensitivity reaction in children with chronic urticaria. Allergy 53: 1074-1077.

Eskenazi B, Kidd SA, Marks AR, et al., (2005): Antioxidant intake is associated with semen 
quality in healthy men. Hum Reprod 20(4):1006-1012.

Eskes TK (1998): Neutral tubes defects, vitamins and homocysteine. European Journal of Pediatrics 157: 5139-5141.

Eweka A and Om'iniabohs F (2011): Histological studies of the effects of monosodium glutamate on the ovaries of adult wistar rats. Ann Med Health Sci Res 1(1):37-43.

Farombi EO and Onyema OO (2006): Monosodium glutamate-induced oxidative damage and genotoxicity in the rat: modulatory role of vitamin C, vitamin E and guercetin. Human Experimental Toxicol 125: 251-259.

Feldman S and Weidenfeld J (2005): Hypothalamic mechanisms mediating glutamate effects on the hypothalamo-pituitary-adrenocortical axis. Journal of Neural Transmission 104(6-7): 633-642.

Feng YH and Hart G (1996): Suppression of oxidant production by diltiazem, nifedipine and verapamil in human neutrophils. Clin Sci 91: 459-466.

Fukuda S, Foster RG, Porter SB, et al., (2002): The antiapoptosis protein surviving is associated with cell cycle entry of normal cord blood CD34+ cells andmodulates cell cycle and proliferation of mouse hematopoietic progenitor cells. Blood 100:2463-2471.

Furst P and Stehle P (2004): What are the essential elements needed for the determination of amino acid requirements in humans? J Nutri 34: 1558 S-1565S.

Gao J, Wu J, Zhao XN, et al., (1994):Transplacental neurotoxic effects of monosodium glutamate on structures and functions of specific brain areas of filial mice. Sheng Li Xue Bao 46: 4451

Geha RS, Beiser A, Ren C, et al., (2000): Review of alleged reaction to monosodium glutamate and outcome of a multicenter double-blind placebo-controlled study. J Nutr 130:1058S$1062 \mathrm{~S}$.

Goldsmith PC (2000): Neuroglial responses to elevated glutamate in the medial basal hypothalamus of the infant mouse. J Nutr 130:1032S-1038S.

Haouzi D, Fourar M, Pellestor F, et al., (2008): Could apoptotic markers help the exploration of men infertility? GynecolObstetFertil., 36: 721-729.

Hsu P-C, Liu M-Y, Hsu C-C, et al., (1998): Effects of vitamin $\mathrm{E}$ and/or $\mathrm{C}$ on reactive oxygen species-related lead toxicity in the rat sperm. Toxicology 128(3):169-179.

Igwebuike UM, Ochiogu IS, Ihedinihu BC, et al., (2011): The effects of oral administration of monosodium glutamate (msg)on the testicular morphologhy and caudaepididymal sperm reserves of young and adult male rats. Vet Arhiv 81(4): 525-534.

Ikonomidou C, Turski L, Stone TW, et al., (1995): Glutamate in Neurodegenerative Disorders. In CNS Neurotransmitters and Neuromodulators: CRC Press 253-272.
Kennedy SM, O'Driscoll L, Purcell R et al., (2003): Prognostic importance of survivin in breast cancer. Br J Cancer 88: 1077-1083.

Konopacka M (2004): Role of vitamin C in oxidative DNA damage. Postepy Hig Med Dosw 58:343-8.

KuriharaK and Kashiwayanagi M (2000): Physiological studies on umami taste. J Nutr 130: 931S-4S.

Lechler P,Wu X, Bernhardt W, et al., (2007):The tumor gene survivin is highly expressed in adult renal tubular cells:implications for a pathophysiological role in the kidney. Am J Pathol 171:1483-1498.

Lenzi A, Gandini L, Picardo M, et al., (2000): Lipoperoxidation damage of spermatozoa polyunsaturated fatty acids (PUFA): scavenger mechanisms and possible scavenger therapies. Front Biosci 5:E1-E15.

Li Y and Schellhorn HE (2007): New developments and novel therapeutic perspectives for vitamin C. J Nutr 137(10):2171-2184.

Lin L, Gu HM, Zhang WN, et al., (1995): Effects of morphine on monosodium glutamate neurotoxicity and its mechanism. Yao XueXueBao30: 806-811 (in Chinese).

Loo BV, Bachschmid M, Spitzer V, et al., (2003): Decreased plasma and tissue levels of vitamin $\mathrm{C}$ in a rat model of aging: implications for antioxidative defense. BiochemBiophys Res Commun 303:483-487

Macho L, Ficková M, Ježová D, et al., (2000): Late effects of postnatal administration of monosodium glutamate on insulin action in adult rats. Physiol. Res 49 (Suppl.1): S79-S85.

Makker K, Agarwal A and Sharma R (2009): Oxidative stress \& male infertility. Indian J Med Res 129(4): 357-367.

Martin LJ, Sieber FE and Traystman RJ (2000): Apoptosis and necrosis occur in separate neuronal populations in hippocampus and cerebellum after ischemia and are associated with differential alterations in metabotropic glutamate receptor signaling pathways. J Cereb Blood Flow Metab 20:153-167.

Marusawa H, Matsuzawa S-I, Welsh K, et al., (2003): HBXIP functions as a cofactor of survivin in apoptosis suppression. EMBO J 22:27292740 .

McCrann DJ, Yang D, Chen H, et al., (2009): Upregulation of Nox4 in theaging vasculature and its association with smooth muscle cell polyploidy. Cell Cycle 8:902-908.

McLachlan RI, O'Donnell L, Stanton PG, et al. (2002): Effects of testosterone plus medroxyprogesterone acetate on semen quality, reproductive hormones, and germ cell populations in normal young men. J Clin Endocrinol Metab 87: 546-56.

McLachlan RI, Wreford NG, O'donnell L, et al., (1996): The endocrine regulation of spermatogenesis: independent roles for testosterone and FSH. J Endocrinol 148: 1-9. 
Mohamed IK (2012): The effects of oral dosage of monosodium glutamate applied for short- and long-terms on histology and ultrastructure of testes of the adult rats. Journal of Animal and Veterinary Advances 11 (1):124-133.

Monno A, Vezzani A, Bastone A, et al., (1995): Extracellular glutamate levels in the hypothalamus and hippocampus of rats after acute or chronic oral intake of monosodium glutamate. Neuroscience Letters 193: 45-48.

Moore KL (2003): Developing Humans. 2nd ed. Philadelphia: W.B. Saunders Co. Ltd. Congenital malformations due to environmental. pp: 173-183.

Mozes S and Sefcikova Z (2004): Obesity and changes of alkaline phosphatase activity in the small intestine of 40 and 80-day old rats subjected to early postnatal overfeeding of monosodium glutamate. Physiol Res 53(2):177-186.

Nakagawa T, Ukai K, Ohyama T, et al., (2000): Effects of chronic administration of sibutramine on body weight, food intake and motor activity in neonatally monosodium glutamate-treated obese female rats: Relatiomhp of antiobesity effect with monoamines. Exp Anim 49: 239249.

Naziroğlu M (2003): Enhanced testicular antioxidant capacity in streptozotocin-induced diabetic rats. Protective role of vitamins $\mathrm{C}$ and $\mathrm{E}$ and selenium.Biol Trace Elem Res 94(1):61-71.

O'shaughnessy PJ, Verhoeven G, De Gendt K, et al., (2010): Direct action through the Sertoli cells is essential for androgen stimulation of spermatogenesis. Endocrinol 151: 2343-2348.

Obochi GO, Malu SP, Abara AE, et al., (2009): Effects of ascorbate on monosodium glutamate- associated toxicities that may impact upon immunocompetence. Toxicological and Enviornmental Chemistry 91(3): 547- 557.

Oforofuo IAO, Onakewhor JUE and Idaewor PE (1997): The effect of chronic administration of MSG on the histology of the adult Wister rat testes: Bioscience Res Comm 9(2): 30.

Onakewhor JUE, Oforofuo IAO and Singh SP (1998): Chronic administration of Monosodium glutamate induces oligozoospermia and glycogen accumulation in Wister rat testes. AfriJ Reprod Health 2(2):190-197.

Onyema OO, Farombi EO, Emerole GO, e al., (2006): Effects of vitamin $\mathrm{E}$ on monosodium glutamate induced hepatotoxicity and oxidative stress in rats. Indian $\mathrm{J}$ BiochemBiophys 43: 20-24.

Ortiz GG, Bitzer-Quintero OK, Beas-Zamte C, et al., (2006): Monosodium glutamate-induced damage in liver and kidney: A morphological and biochemical approach. Biomedecine Pharmacotherapy 60: 86-91.

Pakarainen T, Zhang F, Mäkelä S, et al., (2005): Testosterone replacement therapy induces spermatogenesis and partially restores fertility in luteinizing hormone receptor knockout mice. Endocrinol 146: 596-606.

Reagan- Shaw S, Nihal M and Ahmad N (2008): Dose translation from animal to human studies revisited. The FASEB J. 22 (3): 659- 61.

Rodriquez MC, Obeso JA and Olanow CW (1998): Sub-thalamic nucleus-mediated excitoxicity in Parkinson's disease: A target for neuroprotection. American Journal of Neurology 44: 174-188.

Rogers PP and Blundell JE (1990): Umani and appetite: Effects of Monosodium glutamate on hunger and food intake in human subjects. Physiol Behav 486:801-804.

Sah NK, Khan Z, Khan GJ, et al., (2006): Structural, functional and therapeutic biology of survivin. Cancer Lett 244:164-171.

Samuels A (1999): The toxicity/safety of MSG: A study in suppression of information. Accountability Res 6: 259-310.

Schelman WR, Andres RD, Sipe KJ, et al. (2004): Glutamate mediates cell death and increases the $\mathrm{Bax}$ to $\mathrm{Bcl}-2$ ratio in a differentiated neuronal cell line. Brain Res Mol Brain Res 128: $160-169$.

Scopp A L (1991): MSG and hydrolyzed vegetable protein induced headache: review and case studies. Headache 31: 107-110.

Seo HJ, Ham HD, Jin HY, et al., (2010): Chronic Administration of monosodium Glutamate under Chronic Variable Stress Impaired Hypothalamic- Pituitary-Adrenal Axis Function in Rats. Korean J Physiol Pharmacol 14(4): 213-221.

Seo S-K, Lee H-C, Woo S-H, et al., (2007):Sulindacderived reactive oxygen species induce apoptosis of human multiple myeloma cells via p38 mitogen activated protein kinaseinduced mitochondrial dysfunction. Apoptosis 12:195-209.

Shrilatha B and Muralidhara (2007): Early oxidative stress in testis and epididymal sperm in streptozotocin-induced diabetic mice: its progression and genotoxic consequences. Reprod Toxicol 23(4):578-587.

Singh P, Mann KA, Mangat HK, et al., (2003): Prolonged glutamate excitotoxicity: effects on mitochondrial antioxidants and antioxidant enzymes. Mol Cell Biochem 243(1-2): 139145.

Sönmez M, Türk G and Yüce A (2005): The effects of ascorbic acid supplementation on sperm quality, lipid proxidation and testosterone levels of male Wistar rats. Theriogenology 63(7):2063-2072.

Swelim HH (2004): Monosodium glutamate (MSG) induced retinopathy in adult and neonate CD1 mice. Egypt J Med Lab Sci 13:45-71

Taubert H, Heidenreich C, Holzhausen H-J, et al., (2010): Expression of survivin detected by immunohistochemistry in the cytoplasm and in the nucleus is associated with prognosis of 
leiomyosarcoma and synovial sarcoma patients. BMC Cancer 10:65

Turner TT (2001): The study of varicocele through the use of animal models. Hum Reprod Update 7:78-84.

Urban RJ (1999): Effects of testosterone and growth hormone on muscle function. J Lab Clin Med 134: 7-10.

Walker R and Lupien JR (2000): The safety evaluation of monosodium glutamate. J Nutr $130(4 \mathrm{~S}$ Suppl):1049S-52S.

Wang R, Yeh S, Tzeng C, et al., (2009): Androgen receptor roles in spermatogenesis and fertility: Lessons from testicular cell-specific androgen receptor knockout mice. Endocrinol Rev 30:119-132.

Weikert S, Christoph F, Schulze W, et al., (2006): Testicular expression of survivin and human telomerase reverse transcriptase (hTERT) associated with spermatogenic function in infertile patients. Asian J Androl 8 (1):95100.

Weikert S, Schrader M, Müller M, et al., (2004): Expression of the apoptosis inhibitor survivin in testicular tissue of infertile patients. IntJAndrol27:161-165.

Zai H, Kusano M, Hosaka H, et al., (2009): Monosodium L-glutamate added to a highenergy, high-protein liquid diet promotes gastric emptying. Am J Clin Nutr 89:431-435

Zhang F, Pakrainen T,Poutanen M, et al., (2003): The low gonadotropin-independent constitutive production of testicular testosterone is sufficient to maintain spermatogenesis. Proc. Natl. Acad. Sci. USA. 100, 13692-13697.

Zitzmann M (2008): Effects of testosterone replacement and its pharmacogenetics on physical performance and metabolism. Asian J Androl 10: 364-72.

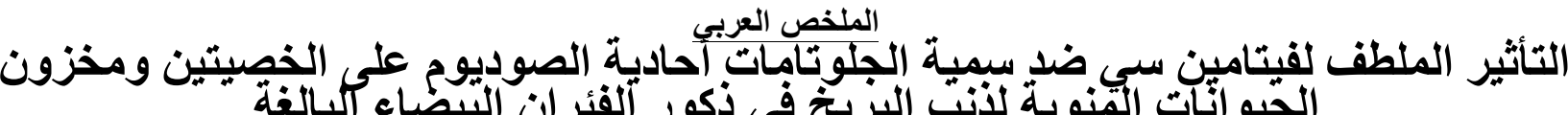

$$
\text { إيناس ابراهيم المداح و منى محمد ابو النور } 1 \text { و منى عبد الحق عبد العظيم². }
$$

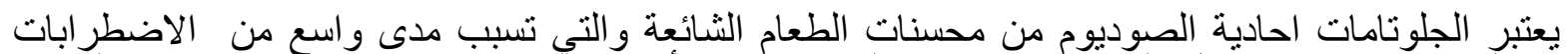

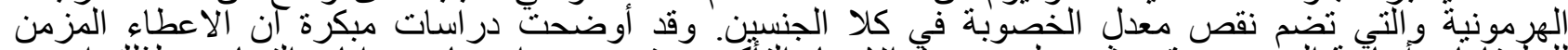

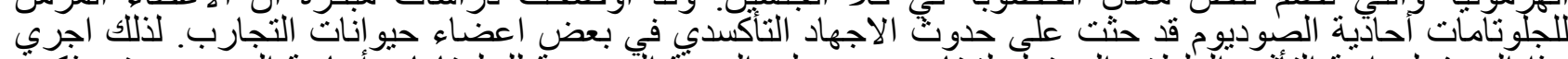

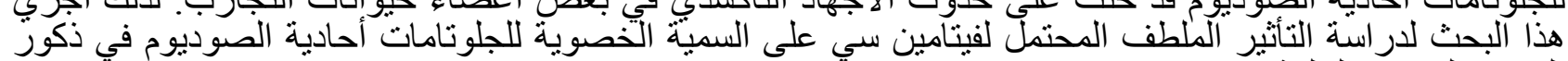

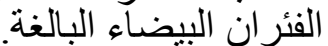

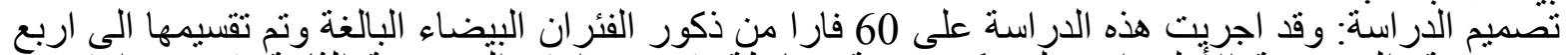

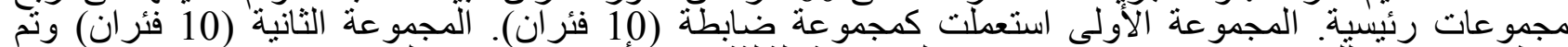

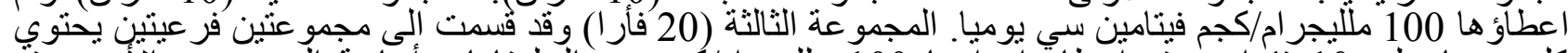

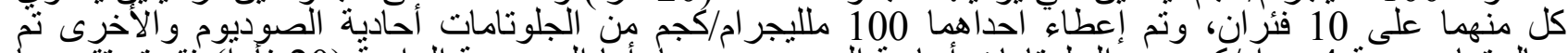

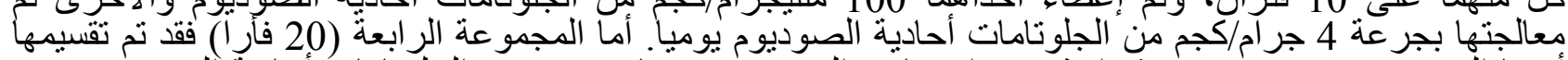

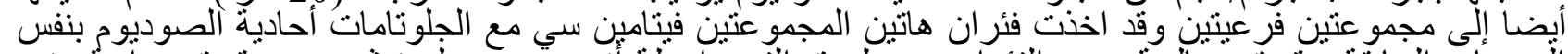

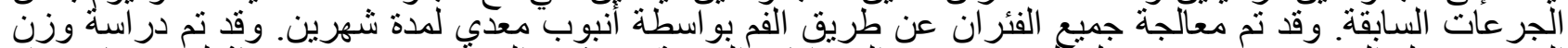

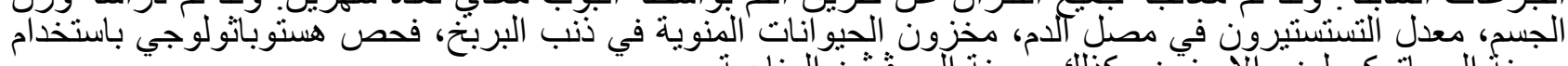

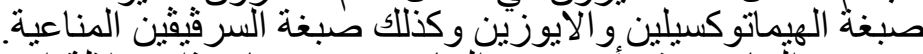

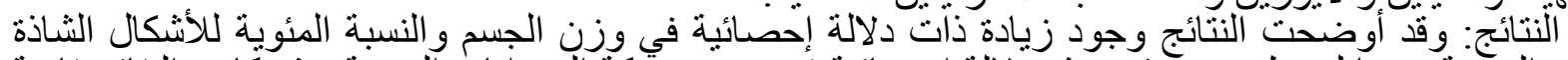

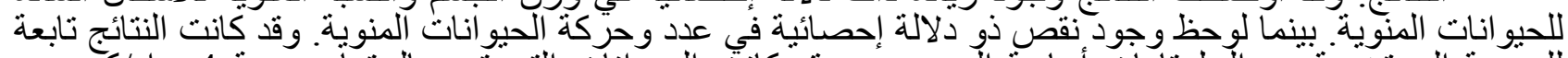

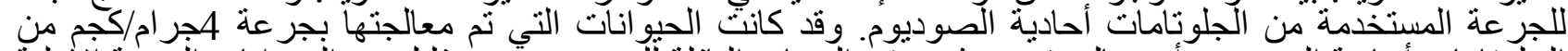

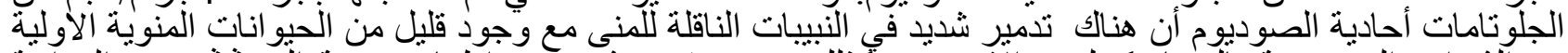

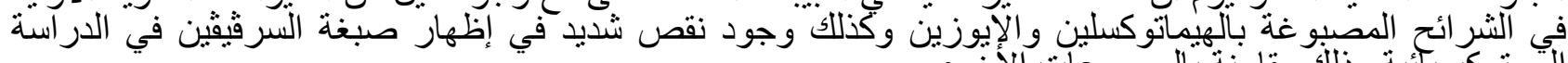

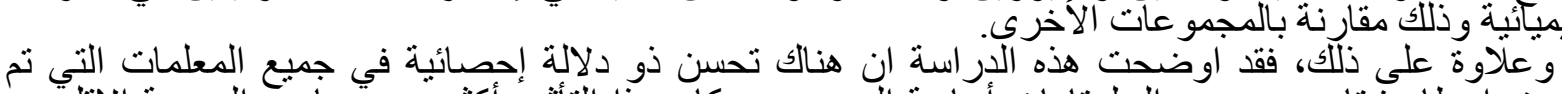

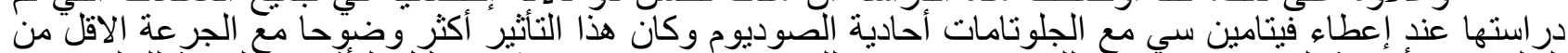

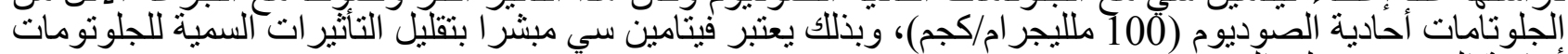
أحادية الصوديوم على الخصيتين. 